Supplement of Atmos. Chem. Phys. Discuss., 15, 13923-13955, 2015

http://www.atmos-chem-phys-discuss.net/15/13923/2015/

doi:10.5194/acpd-15-13923-2015-supplement

(C) Author(s) 2015. CC Attribution 3.0 License.

(c) (i)

Supplement of

\title{
A better understanding of hydroxyl radical photochemical sources in cloud waters collected at the puy de Dôme station: experimental vs. modeled formation rates
}

\section{A. Bianco et al.}

Correspondence to: M. Brigante (marcello.brigante@univ-bpclermont.fr)

and L. Deguillaume (1.deguillaume@opgc.univ-bpclermont.fr)

The copyright of individual parts of the supplement might differ from the CC-BY 3.0 licence. 


\begin{tabular}{|c|c|c|c|c|c|}
\hline Reactions & & $\begin{array}{r}k_{298} \\
\left(M^{-n+1} s^{-1}\right)\end{array}$ & $\begin{array}{c}\mathrm{Ea} / \mathrm{R} \\
(\mathrm{K})\end{array}$ & References & Notes \\
\hline \multicolumn{6}{|l|}{$\mathrm{HO}_{\mathrm{x}}$ chemistry } \\
\hline $\mathrm{O}_{3}+\mathrm{hv} \rightarrow \mathrm{H}_{2} \mathrm{O}_{2}+\mathrm{O}_{2}-\mathrm{H}_{2} \mathrm{O}$ & $R(1)$ & Calculated & & $\begin{array}{l}\text { (Graedel and } \\
\text { Weschler, 1981) }\end{array}$ & \\
\hline $\mathrm{H}_{2} \mathrm{O}_{2}+\mathrm{hv} \rightarrow 2 \mathrm{HO}^{\circ}$ & $R(2)$ & Calculated & & $\begin{array}{l}\text { (Graedel and } \\
\text { Weschler, 1981) }\end{array}$ & \\
\hline $\mathrm{HO}_{2}^{\cdot}+\mathrm{HO}_{2}^{\cdot} \rightarrow \mathrm{H}_{2} \mathrm{O}_{2}+\mathrm{O}_{2}$ & $R(3)$ & $8.310^{5}$ & 2700 & (Bielski et al., 1985) & \\
\hline $\mathrm{HO}_{2}{ }^{\circ}+\mathrm{O}_{2}{ }^{-} \rightarrow \mathrm{H}_{2} \mathrm{O}_{2}+\mathrm{O}_{2}+\mathrm{OH}^{-}-\mathrm{H}_{2} \mathrm{O}$ & $R(4)$ & $9.610^{7}$ & 910 & $\begin{array}{l}\text { (Christensen and } \\
\text { Sehested, 1988) }\end{array}$ & \\
\hline $\mathrm{O}_{3}+\mathrm{HO}_{2}^{\cdot} \rightarrow \mathrm{HO}^{\bullet}+2 \mathrm{O}_{2}$ & $R(5)$ & $<1.010^{4}$ & & (Sehested et al., 1984) & 1 \\
\hline $\mathrm{O}_{3}+\mathrm{O}_{2}^{--} \rightarrow \mathrm{HO}^{\circ}+2 \mathrm{O}_{2}+\mathrm{OH}^{-}-\mathrm{H}_{2} \mathrm{O}$ & $R(6)$ & $1.510^{9}$ & 2200 & (Sehested et al., 1983) & \\
\hline \multicolumn{6}{|l|}{ Iron chemistry } \\
\hline $\mathrm{Fe}^{3+}+\mathrm{hv} \rightarrow \mathrm{Fe}^{2+}+\mathrm{HO}^{\circ}+\mathrm{H}^{+}-\mathrm{H}_{2} \mathrm{O}$ & $R(7)$ & Calculated & & $\begin{array}{l}\text { (Benkelberg and } \\
\text { Warneck, 1995) }\end{array}$ & \\
\hline$[\mathrm{Fe}(\mathrm{OH})]^{2+}+\mathrm{hv} \rightarrow \mathrm{Fe}^{2+}+\mathrm{HO}^{\bullet}$ & $R(8)$ & Calculated & & $\begin{array}{l}\text { (Benkelberg and } \\
\text { Warneck, 1995) }\end{array}$ & \\
\hline$\left[\mathrm{Fe}(\mathrm{OH})_{2}\right]^{+}+\mathrm{hv} \rightarrow \mathrm{Fe}^{2+}+\mathrm{HO}^{\circ}+\mathrm{OH}^{-}$ & $R(9)$ & Calculated & & $\begin{array}{l}\text { (Benkelberg et al., } \\
\text { 1991); (Weschler et } \\
\text { al., 1986) }\end{array}$ & \\
\hline$\left[\mathrm{Fe}\left(\mathrm{SO}_{4}\right)\right]^{+}+\mathrm{hv} \rightarrow \mathrm{Fe}^{2+}+\mathrm{SO}_{4}^{-}$ & $R(10)$ & Calculated & & $\begin{array}{l}\text { (Benkelberg and } \\
\text { Warneck, 1995) }\end{array}$ & \\
\hline $\mathrm{H}_{2} \mathrm{O}_{2}+\mathrm{Fe}^{2+} \rightarrow \mathrm{Fe}^{3+}+\mathrm{HO}^{\circ}+\mathrm{OH}^{-}$ & $R(11)$ & $5.210^{1}$ & 5050 & $\begin{array}{l}\text { (Christensen et al., } \\
\text { 1993); (Kremer, 2003) }\end{array}$ & \\
\hline $\mathrm{H}_{2} \mathrm{O}_{2}+\mathrm{FeO}^{2+} \rightarrow \mathrm{Fe}^{3+}+\mathrm{HO}_{2}^{-}+\mathrm{OH}^{-}$ & $R(12)$ & $9.510^{3}$ & 2800 & (Jacobsen et al., 1997) & \\
\hline $\mathrm{O}_{2}{ }^{--}+\mathrm{Fe}^{2+} \rightarrow \mathrm{Fe}^{3+}+\mathrm{H}_{2} \mathrm{O}_{2}-2 \mathrm{H}^{+}$ & $R(13)$ & $1.010^{7}$ & & $\begin{array}{l}\text { (Rush and Bielski, } \\
\text { 1985) }\end{array}$ & \\
\hline $\mathrm{O}_{2}^{\cdot-}+\mathrm{Fe}^{3+} \rightarrow \mathrm{Fe}^{2+}+\mathrm{O}_{2}$ & $R(14)$ & $1.510^{8}$ & & & $=\mathrm{k}\left(\mathrm{O}_{2}{ }^{-}+[\mathrm{Fe}(\mathrm{OH})]^{2+}\right)$ \\
\hline $\mathrm{O}_{2}{ }^{\circ-}+[\mathrm{Fe}(\mathrm{OH})]^{2+} \rightarrow \mathrm{Fe}^{2+}+\mathrm{O}_{2}+\mathrm{OH}^{-}$ & $R(15)$ & $1.510^{8}$ & & $\begin{array}{l}\text { (Rush and Bielski, } \\
\text { 1985) }\end{array}$ & \\
\hline $\mathrm{O}_{2}{ }^{--}+\left[\mathrm{Fe}(\mathrm{OH})_{2}\right]^{+} \rightarrow \mathrm{Fe}^{2+}+\mathrm{O}_{2}+2 \mathrm{OH}^{-}$ & $R(16)$ & $1.510^{8}$ & & & $=\mathrm{k}\left(\mathrm{O}_{2}{ }^{-}+[\mathrm{Fe}(\mathrm{OH})]^{2+}\right)$ \\
\hline $\mathrm{HO}_{2}{ }^{-}+\mathrm{Fe}^{2+} \rightarrow \mathrm{Fe}^{3+}+\mathrm{H}_{2} \mathrm{O}_{2}-\mathrm{H}^{+}$ & $R(17)$ & $1.210^{6}$ & 5050 & (Jayson et al., 1973a) & \\
\hline $\mathrm{HO}_{2}{ }^{\circ}+\mathrm{FeO}^{2+} \rightarrow \mathrm{Fe}^{3+}+\mathrm{O}_{2}+\mathrm{OH}^{-}$ & $R(18)$ & $2.010^{6}$ & & (Jacobsen et al., 1997) & \\
\hline $\mathrm{O}_{3}+\mathrm{Fe}^{2+} \rightarrow \mathrm{FeO}^{2+}+\mathrm{O}_{2}$ & $R(19)$ & $8.210^{5}$ & & (Logager et al., 1992) & \\
\hline $\mathrm{FeO}^{2+} \rightarrow \mathrm{Fe}^{3+}+\mathrm{HO}^{\circ}+\mathrm{OH}^{-}-\mathrm{H}_{2} \mathrm{O}$ & $R(20)$ & $1.310^{-2}$ & 4100 & (Jacobsen et al., 1997) & \\
\hline $\mathrm{FeO}^{2+}+\mathrm{Fe}^{2+} \rightarrow 2 \mathrm{Fe}^{3+}+2 \mathrm{OH}^{-}-\mathrm{H}_{2} \mathrm{O}$ & $R(21)$ & $7.210^{4}$ & 840 & (Jacobsen et al., 1997) & \\
\hline $\mathrm{FeO}^{2+}+\mathrm{Fe}^{2+} \rightarrow \mathrm{Fe}(\mathrm{OH})_{2} \mathrm{Fe}^{4+}-\mathrm{H}_{2} \mathrm{O}$ & $R(22)$ & $1.810^{4}$ & 5050 & (Jacobsen et al., 1997) & \\
\hline $\mathrm{Fe}(\mathrm{OH})_{2} \mathrm{Fe}^{4+} \rightarrow 2 \mathrm{Fe}^{3+}+2 \mathrm{OH}^{-}$ & $R(23)$ & $4.910^{-1}$ & 8800 & (Jacobsen et al., 1997) & \\
\hline $\mathrm{Fe}(\mathrm{OH})_{2} \mathrm{Fe}^{4+}+\mathrm{H}^{+} \rightarrow 2 \mathrm{Fe}^{3+}+2 \mathrm{H}_{2} \mathrm{O}-\mathrm{H}^{+}$ & $R(24)$ & 2.0 & 5650 & (Jacobsen et al., 1997) & \\
\hline $\mathrm{Cl}_{2}^{\circ-}+\mathrm{Fe}^{2+} \rightarrow \mathrm{Fe}^{3+}+2 \mathrm{Cl}^{-}$ & $R(25)$ & $1.010^{7}$ & 3060 & $\begin{array}{l}\text { (Thornton and } \\
\text { Laurence, 1973) }\end{array}$ & \\
\hline $\mathrm{Cl}_{2}{ }^{--}+\mathrm{Fe}^{2+} \rightarrow[\mathrm{FeCl}]^{2+}+\mathrm{Cl}^{-}$ & $R(26)$ & $4.010^{6}$ & 3700 & (Thornton and & \\
\hline
\end{tabular}




\begin{tabular}{|c|c|c|c|c|c|}
\hline Reactions & & $\begin{array}{l}k_{298} \\
\left(M^{-n+1} s^{-1}\right)\end{array}$ & $\begin{array}{c}\mathrm{Ea} / \mathrm{R} \\
(\mathrm{K})\end{array}$ & References & Notes \\
\hline $\mathrm{Cl}^{-}+\mathrm{Fe}(\mathrm{O})^{2+} \rightarrow \mathrm{Fe}^{3+}+\mathrm{ClOH}^{-}-\mathrm{H}^{+}$ & $R(27)$ & $1.010^{2}$ & & $\begin{array}{l}\text { Laurence, 1973) } \\
\text { (Jacobsen et al., 1998) }\end{array}$ & \\
\hline $\mathrm{NO}_{3}{ }^{-}+\mathrm{Fe}^{2+} \rightarrow \mathrm{Fe}^{3+}+\mathrm{NO}_{3}^{-}$ & $R(28)$ & $8.010^{6}$ & & (Pikaev et al., 1974) & \\
\hline $\mathrm{NO}_{2}+\mathrm{Fe}^{2+} \rightarrow \mathrm{Fe}^{3+}+\mathrm{NO}_{2}^{-}$ & $R(29)$ & $3.110^{4}$ & & (Epstein et al., 1982) & \\
\hline $\mathrm{HNO}_{2}+\mathrm{FeO}^{2+} \rightarrow \mathrm{Fe}^{3+}+\mathrm{NO}_{2}+\mathrm{OH}^{-}$ & $R(30)$ & $1.110^{4}$ & 4150 & (Jacobsen et al., 1998) & \\
\hline $\mathrm{NO}_{2}^{-}+\mathrm{FeO}^{2+} \rightarrow \mathrm{Fe}^{3+}+\mathrm{NO}_{2}+\mathrm{OH}^{-}-\mathrm{H}^{+}$ & $R(31)$ & $<1.010^{5}$ & & (Jacobsen et al., 1998) & \\
\hline $\mathrm{HSO}_{3}{ }^{-}+[\mathrm{Fe}(\mathrm{OH})]^{2+} \rightarrow \mathrm{Fe}^{2+}+\mathrm{SO}_{3}{ }^{\circ-}-\mathrm{H}_{2} \mathrm{O}$ & $R(32)$ & $3.010^{1}$ & & (Ziajka et al., 1994) & \\
\hline $\mathrm{SO}_{5}{ }^{\circ-}+\mathrm{Fe}^{2+} \rightarrow[\mathrm{Fe}(\mathrm{OH})]^{2+}+\mathrm{HSO}_{5}^{-}-\mathrm{H}_{2} \mathrm{O}$ & $R(33)$ & $2.610^{7}$ & & (Williams, 1996) & \\
\hline $\mathrm{HSO}_{5}^{-}+\mathrm{Fe}^{2+} \rightarrow[\mathrm{Fe}(\mathrm{OH})]^{2+}+\mathrm{SO}_{4}^{\circ-}$ & $R(34)$ & $3.010^{4}$ & & $\begin{array}{l}\text { (Gilbert and Stell, } \\
\text { 1990) }\end{array}$ & \\
\hline $\mathrm{SO}_{4}{ }^{--}+\mathrm{Fe}^{2+} \rightarrow \mathrm{Fe}^{3+}+\mathrm{SO}_{4}^{2-}$ & $R(35)$ & $4.110^{9}$ & -2165 & (Buxton et al., 1997) & \\
\hline $\mathrm{O}_{2}{ }^{--}+\left[\mathrm{Fe}\left(\mathrm{SO}_{4}\right)\right]^{+} \rightarrow \mathrm{Fe}^{2+}+\mathrm{SO}_{4}^{2-}+\mathrm{O}_{2}$ & $R(36)$ & $1.510^{8}$ & & $\begin{array}{l}\text { (Rush and Bielski, } \\
\text { 1985) }\end{array}$ & \\
\hline $\mathrm{S}_{2} \mathrm{O}_{8}^{2-}+\mathrm{Fe}^{2+} \rightarrow \mathrm{Fe}^{3+}+\mathrm{SO}_{4}^{--}+\mathrm{SO}_{4}^{2-}$ & $R(37)$ & $1.710^{1}$ & & (Buxton et al., 1997) & \\
\hline $\mathrm{HSO}_{3}{ }^{-}+\mathrm{FeO}^{2+} \rightarrow \mathrm{Fe}^{3+}+\mathrm{SO}_{3}{ }^{-}+\mathrm{OH}^{-}$ & $R(38)$ & $2.510^{5}$ & & (Jacobsen et al., 1998) & \\
\hline $\mathrm{Fe}^{3+}+\mathrm{SO}_{4}^{2-} \rightarrow\left[\mathrm{Fe}\left(\mathrm{SO}_{4}\right)\right]^{+}$ & $R(39)$ & $3.210^{3}$ & & (Jayson et al., 1973b) & \\
\hline$\left[\mathrm{Fe}\left(\mathrm{SO}_{4}\right)\right]^{+} \rightarrow \mathrm{Fe}^{3+}+\mathrm{SO}_{4}^{2-}$ & $R(40)$ & $2.710^{1}$ & & (Jayson et al., 1973b) & \\
\hline $\mathrm{Fe}^{3+}+\mathrm{Cl}^{-} \rightarrow[\mathrm{FeCl}]^{2+}$ & $R(41)$ & 4.8 & & (Xu et al., 1985) & \\
\hline$[\mathrm{FeCl}]^{2+} \rightarrow \mathrm{Fe}^{3+}+\mathrm{Cl}^{-}$ & $R(42)$ & $9.210^{-1}$ & & $\begin{array}{l}\text { Estimated following } \\
\text { (Nadtochenko and } \\
\text { Kiwi, 1998) }\end{array}$ & 2 \\
\hline $\mathrm{C}_{2} \mathrm{O}_{4}^{--}+\mathrm{O}_{2} \rightarrow 2 \mathrm{CO}_{2}+\mathrm{O}_{2}^{-\cdot}$ & $R(43)$ & $2.410^{9}$ & & $\begin{array}{l}\text { (Hislop and Bolton, } \\
\text { 1999) }\end{array}$ & \\
\hline $\mathrm{CO}\left(\mathrm{O}^{-}\right) \mathrm{CO}\left(\mathrm{O}^{-}\right)+\mathrm{Fe}^{3+} \rightarrow\left[\mathrm{Fe}\left(\mathrm{C}_{2} \mathrm{O}_{4}\right)\right]^{+}$ & $R(44)$ & $7.510^{6}$ & & & 3 \\
\hline$\left[\mathrm{Fe}\left(\mathrm{C}_{2} \mathrm{O}_{4}\right)\right]^{+} \rightarrow \mathrm{CO}\left(\mathrm{O}^{-}\right) \mathrm{CO}\left(\mathrm{O}^{-}\right)+\mathrm{Fe}^{3+}$ & $R(45)$ & $3.010^{-3}$ & & $\begin{array}{l}\text { (Moorhead and Sutin, } \\
\text { 1966) }\end{array}$ & 4 \\
\hline $\mathrm{CO}\left(\mathrm{O}^{-}\right) \mathrm{CO}\left(\mathrm{O}^{-}\right)+\left[\mathrm{Fe}\left(\mathrm{C}_{2} \mathrm{O}_{4}\right)\right]^{+} \rightarrow\left[\mathrm{Fe}\left(\mathrm{C}_{2} \mathrm{O}_{4}\right)_{2}\right]^{-}$ & $R(46)$ & $1.910^{4}$ & & & 3 \\
\hline$\left[\mathrm{Fe}\left(\mathrm{C}_{2} \mathrm{O}_{4}\right)_{2}\right]^{-} \rightarrow \mathrm{CO}\left(\mathrm{O}^{-}\right) \mathrm{CO}\left(\mathrm{O}^{-}\right)+\left[\mathrm{Fe}\left(\mathrm{C}_{2} \mathrm{O}_{4}\right)\right]^{+}$ & $R(47)$ & $3.010^{-3}$ & & & $\begin{array}{l}=k\left(\left[\mathrm{Fe}\left(\mathrm{C}_{2} \mathrm{O}_{4}\right)\right]^{+} \rightarrow\right. \\
\left.\mathrm{CO}\left(\mathrm{O}^{-}\right) \mathrm{CO}\left(\mathrm{O}^{-}\right)+\mathrm{Fe}^{3+}\right)\end{array}$ \\
\hline $\mathrm{CO}\left(\mathrm{O}^{-}\right) \mathrm{CO}\left(\mathrm{O}^{-}\right)+\left[\mathrm{Fe}\left(\mathrm{C}_{2} \mathrm{O}_{4}\right)_{2}\right]^{-} \rightarrow\left[\mathrm{Fe}\left(\mathrm{C}_{2} \mathrm{O}_{4}\right)_{3}\right]^{3-}$ & $R(48)$ & $4.810^{1}$ & & & 3 \\
\hline$\left[\mathrm{Fe}\left(\mathrm{C}_{2} \mathrm{O}_{4}\right)_{3}\right]^{3-} \rightarrow \mathrm{CO}\left(\mathrm{O}^{-}\right) \mathrm{CO}\left(\mathrm{O}^{-}\right)+\left[\mathrm{Fe}\left(\mathrm{C}_{2} \mathrm{O}_{4}\right)_{2}\right]^{-}$ & $R(49)$ & $3.010^{-3}$ & & & $\begin{array}{l}=k\left(\left[\mathrm{Fe}\left(\mathrm{C}_{2} \mathrm{O}_{4}\right)\right]^{+} \rightarrow\right. \\
\left.\mathrm{CO}\left(\mathrm{O}^{-}\right) \mathrm{CO}\left(\mathrm{O}^{-}\right)+\mathrm{Fe}^{3+}\right)\end{array}$ \\
\hline$\left[\mathrm{Fe}\left(\mathrm{C}_{2} \mathrm{O}_{4}\right)\right]^{+}+\mathrm{hv} \rightarrow \mathrm{Fe}^{2+}+\mathrm{C}_{2} \mathrm{O}_{4}^{\cdot-}$ & $R(50)$ & Calculated & & (Long et al., 2013) & \\
\hline$\left[\mathrm{Fe}\left(\mathrm{C}_{2} \mathrm{O}_{4}\right)_{2}\right]^{-}+\mathrm{hv} \rightarrow \mathrm{Fe}^{2+}+\mathrm{CO}\left(\mathrm{O}^{-}\right) \mathrm{CO}\left(\mathrm{O}^{-}\right)+\mathrm{C}_{2} \mathrm{O}_{4}^{*-}$ & $R(51)$ & Calculated & & $\begin{array}{l}\text { (Faust and Zepp, } \\
\text { 1993) }\end{array}$ & \\
\hline$\left[\mathrm{Fe}\left(\mathrm{C}_{2} \mathrm{O}_{4}\right)_{3}\right]^{3-}+\mathrm{hv} \rightarrow \mathrm{Fe}^{2+}+2 \mathrm{CO}\left(\mathrm{O}^{-}\right) \mathrm{CO}\left(\mathrm{O}^{-}\right)+\mathrm{C}_{2} \mathrm{O}_{4}{ }^{--}$ & $R(52)$ & Calculated & & $\begin{array}{l}\text { (Faust and Zepp, } \\
\text { 1993) }\end{array}$ & \\
\hline \multicolumn{6}{|l|}{ Chlorine chemistry } \\
\hline $\mathrm{Cl}^{*} \rightarrow \mathrm{OHCl}^{-}+\mathrm{H}^{+}-\mathrm{H}_{2} \mathrm{O}$ & $R(53)$ & $1.710^{5}$ & & $(Y u, 2004)$ & \\
\hline
\end{tabular}




\begin{tabular}{|c|c|c|c|c|c|}
\hline Reactions & & $\begin{array}{c}k_{298} \\
\left(M^{-n+1} s^{-1}\right)\end{array}$ & $\begin{array}{c}\mathrm{Ea} / \mathrm{R} \\
(\mathrm{K})\end{array}$ & References & Notes \\
\hline $\mathrm{OHCl}^{-}+\mathrm{H}^{+} \rightarrow \mathrm{Cl}^{\bullet}+\mathrm{H}_{2} \mathrm{O}$ & $R(54)$ & $3.310^{10}$ & & (Yu and Barker, 2003) & \\
\hline $\mathrm{Cl}^{\circ}+\mathrm{Cl}^{-} \rightarrow \mathrm{Cl}_{2}^{\cdot-}$ & $R(55)$ & $7.810^{9}$ & & (Yu and Barker, 2003) & \\
\hline $\mathrm{Cl}_{2}{ }^{\circ} \rightarrow \mathrm{Cl}^{\circ}+\mathrm{Cl}^{-}$ & $R(56)$ & $5.710^{4}$ & & (Yu and Barker, 2003) & \\
\hline $\mathrm{Cl}_{2}{ }^{-} \rightarrow \mathrm{OHCl}^{-}+\mathrm{Cl}^{-}+\mathrm{H}^{+}-\mathrm{H}_{2} \mathrm{O}$ & $R(57)$ & $1.3 .10^{3}$ & & $(Y u, 2004)$ & \\
\hline $\mathrm{Cl}_{2}^{--}+\mathrm{OH}^{-} \rightarrow \mathrm{OHCl}^{-}+\mathrm{Cl}^{-}$ & $R(58)$ & $2.010^{7}$ & & (Grigor'ev et al., 1987) & \\
\hline $\mathrm{OHCl}^{-}+\mathrm{Cl}^{-} \rightarrow \mathrm{Cl}_{2}{ }^{\circ-}+\mathrm{OH}^{-}$ & $R(59)$ & $1.010^{4}$ & & (Grigor'ev et al., 1987) & \\
\hline $\mathrm{Cl}_{2}{ }^{-}+\mathrm{Cl}_{2}{ }^{--} \rightarrow \mathrm{Cl}_{2}+2 \mathrm{Cl}^{-}$ & $R(60)$ & $9.010^{8}$ & & $(Y u, 2004)$ & \\
\hline $\mathrm{Cl}_{2}^{-}+\mathrm{Cl}^{\circ} \rightarrow \mathrm{Cl}_{2}+\mathrm{Cl}^{-}$ & $R(61)$ & $2.110^{9}$ & & (Yu, 2004) & \\
\hline $\mathrm{Cl}_{2} \rightarrow \mathrm{Cl}^{-}+\mathrm{HOCl}+\mathrm{H}^{+}-\mathrm{H}_{2} \mathrm{O}$ & $R(62)$ & $2.210^{1}$ & 7600 & $\begin{array}{l}\text { (Wang and } \\
\text { Margerum, 1994) }\end{array}$ & \\
\hline $\mathrm{Cl}^{-}+\mathrm{HOCl}+\mathrm{H}^{+} \rightarrow \mathrm{Cl}_{2}+\mathrm{H}_{2} \mathrm{O}$ & $R(63)$ & $2.110^{4}$ & 3500 & $\begin{array}{l}\text { (Wang and } \\
\text { Margerum, 1994) }\end{array}$ & \\
\hline $\mathrm{HOCl}+\mathrm{HO}_{2}^{\cdot} \rightarrow \mathrm{Cl}^{\cdot}+\mathrm{O}_{2}+\mathrm{H}_{2} \mathrm{O}$ & $R(64)$ & $7.510^{6}$ & & & $=\mathrm{k}\left(\mathrm{HOCl}+\mathrm{O}_{2}{ }^{*}\right)$ \\
\hline $\mathrm{HOCl}+\mathrm{O}_{2}^{--} \rightarrow \mathrm{Cl}^{\circ}+\mathrm{O}_{2}+\mathrm{OH}^{-}$ & $R(65)$ & $7.510^{6}$ & & $\begin{array}{l}\text { (Long and Bielski, } \\
\text { 1980) }\end{array}$ & \\
\hline $\mathrm{Cl}_{2}+\mathrm{HO}_{2}^{\cdot} \rightarrow \mathrm{Cl}_{2}^{-{ }^{-}}+\mathrm{O}_{2}+\mathrm{H}^{+}$ & $R(66)$ & $1.010^{9}$ & & $\begin{array}{l}\text { (Bjergbakke et al., } \\
\text { 1981) }\end{array}$ & \\
\hline $\mathrm{Cl}_{2}+\mathrm{O}_{2}^{-{ }^{-}} \rightarrow \mathrm{Cl}_{2}{ }^{--}+\mathrm{O}_{2}$ & $R(67)$ & $1.010^{9}$ & & & $=\mathrm{k}\left(\mathrm{Cl}_{2}+\mathrm{HO}_{2}^{\cdot}\right)$ \\
\hline $\mathrm{HO}_{2}{ }^{-}+\mathrm{Cl}^{\cdot} \rightarrow \mathrm{Cl}^{-}+\mathrm{O}_{2}+\mathrm{H}^{+}$ & $R(68)$ & $3.110^{9}$ & 1500 & $\begin{array}{l}\text { (Graedel and } \\
\text { Goldberg, 1983) }\end{array}$ & \\
\hline $\mathrm{H}_{2} \mathrm{O}_{2}+\mathrm{Cl}^{\cdot} \rightarrow \mathrm{Cl}^{-}+\mathrm{HO}_{2}^{\cdot}+\mathrm{H}^{+}$ & $R(69)$ & $2.010^{9}$ & & (Yu and Barker, 2003) & \\
\hline $\mathrm{Cl}^{-}+\mathrm{NO}_{3}{ }^{-} \rightarrow \mathrm{NO}_{3}^{-}+\mathrm{Cl}^{-}$ & $R(70)$ & $1.010^{7}$ & 4300 & (Exner et al., 1992) & \\
\hline $\mathrm{NO}_{3}^{-}+\mathrm{Cl}^{\circ} \rightarrow \mathrm{Cl}^{-}+\mathrm{NO}_{3}^{-}$ & $R(71)$ & $1.010^{8}$ & & (Buxton et al., 2000) & \\
\hline $\mathrm{SO}_{4}{ }^{--}+\mathrm{Cl}^{-}+\rightarrow \mathrm{SO}_{4}^{2-}+\mathrm{Cl}^{\circ}$ & $R(72)$ & $2.510^{8}$ & & (Buxton et al., 1999) & \\
\hline $\mathrm{SO}_{4}^{2-}+\mathrm{Cl}^{\cdot} \rightarrow \mathrm{SO}_{4}^{-{ }^{-}}+\mathrm{Cl}^{-}$ & $R(73)$ & $2.110^{8}$ & & (Buxton et al., 1999) & \\
\hline $\mathrm{HO}_{2}{ }^{-}+\mathrm{Cl}_{2}{ }^{-} \rightarrow \mathrm{O}_{2}+2 \mathrm{Cl}^{-}+\mathrm{H}^{+}$ & $R(74)$ & $1.310^{10}$ & & (Jacobi et al., 1996) & \\
\hline $\mathrm{O}_{2}^{\circ-}+\mathrm{Cl}_{2}^{\circ-} \rightarrow \mathrm{O}_{2}+2 \mathrm{Cl}^{-}$ & $R(75)$ & $6.010^{9}$ & & (Jacobi et al., 1996) & \\
\hline $\mathrm{H}_{2} \mathrm{O}_{2}+\mathrm{Cl}_{2}^{\cdot-} \rightarrow 2 \mathrm{Cl}^{-}+\mathrm{HO}_{2}^{\cdot}+\mathrm{H}^{+}$ & $R(76)$ & $6.210^{6}$ & & $(Y u, 2004)$ & \\
\hline $\mathrm{O}_{3}+\mathrm{Cl}_{2}^{\circ-} \rightarrow \mathrm{OHCl}+\mathrm{Cl}^{\circ}+\mathrm{O}_{2}+\mathrm{OH}^{-}-\mathrm{H}_{2} \mathrm{O}$ & $R(77)$ & $9.010^{7}$ & & (Bielski, 1993) & \\
\hline $\mathrm{HSO}_{3}^{-}+\mathrm{Cl}_{2}^{-} \rightarrow \mathrm{SO}_{3}^{--}+2 \mathrm{Cl}^{-}+\mathrm{H}^{+}$ & $R(78)$ & $1.710^{8}$ & 400 & (Jacobi et al., 1996) & \\
\hline $\mathrm{SO}_{3}^{2{ }^{2-}}+\mathrm{Cl}_{2}{ }^{--} \rightarrow \mathrm{SO}_{3}{ }^{\circ-}+2 \mathrm{Cl}^{-}$ & $R(79)$ & $6.210^{7}$ & & (Jacobi et al., 1996) & \\
\hline \multicolumn{6}{|l|}{ Carbonate chemistry } \\
\hline $\mathrm{HCO}_{3}^{-}+\mathrm{SO}_{4}^{-{ }^{-}} \rightarrow \mathrm{SO}_{4}^{2-}+\mathrm{CO}_{3}{ }^{--}+\mathrm{H}^{+}$ & $R(80)$ & $2.810^{6}$ & 2100 & \multirow{6}{*}{\multicolumn{2}{|c|}{$\begin{array}{l}\text { (Huie and Clifton, } \\
\text { 1990) } \\
\text { (Padmaja et al., 1993) } \\
\text { (Buxton et al., 2000) } \\
\text { (Mertens and Von } \\
\text { Sonntag, 1995) } \\
\text { (Huie and Clifton, } \\
\text { 1990) } \\
\quad 4\end{array}$}} \\
\hline $\mathrm{CO}_{3}^{2-}+\mathrm{SO}_{4}^{--} \rightarrow \mathrm{SO}_{4}^{2-}+\mathrm{CO}_{3}^{\cdot-}$ & $R(81)$ & $4.110^{6}$ & 3200 & & \\
\hline $\mathrm{HCO}_{3}^{-}+\mathrm{Cl}^{-} \rightarrow \mathrm{Cl}^{-}+\mathrm{CO}_{3}{ }^{-}+\mathrm{H}^{+}$ & $R(82)$ & $2.410^{9}$ & & & \\
\hline $\mathrm{CO}_{3}^{2-}+\mathrm{Cl}^{\cdot} \rightarrow \mathrm{Cl}^{-}+\mathrm{CO}_{3}^{\circ-}$ & $R(83)$ & $5.010^{8}$ & & & \\
\hline $\mathrm{CO}_{3}{ }^{-*}+\mathrm{CO}_{3}{ }^{-*} \rightarrow 2 \mathrm{O}_{2}{ }^{*}+2 \mathrm{CO}_{2}-\mathrm{O}_{2}$ & $R(84)$ & $2.210^{6}$ & & & \\
\hline & & & & & \\
\hline
\end{tabular}




\begin{tabular}{|c|c|c|c|c|c|}
\hline Reactions & & $\begin{array}{c}k_{298} \\
\left(M^{-n+1} s^{-1}\right)\end{array}$ & $\begin{array}{c}\mathrm{Ea} / \mathrm{R} \\
(\mathrm{K})\end{array}$ & References & Notes \\
\hline $\mathrm{CO}_{3}{ }^{--}+\mathrm{HO}_{2}^{\cdot} \rightarrow \mathrm{HCO}_{3}^{-}+\mathrm{O}_{2}$ & $R(85)$ & $5.610^{7}$ & & (Behar et al., 1970) & \\
\hline $\mathrm{CO}_{3}^{\cdot-}+\mathrm{O}_{2}^{\cdot-} \rightarrow \mathrm{CO}_{3}^{2-}+\mathrm{O}_{2}$ & $R(86)$ & $6.510^{8}$ & & (Eriksen et al., 1985) & \\
\hline $\mathrm{CO}_{3}{ }^{-}+\mathrm{H}_{2} \mathrm{O}_{2} \rightarrow \mathrm{HO}_{2}^{-}+\mathrm{HCO}_{3}^{-}$ & $R(87)$ & $4.310^{5}$ & & (Draganic et al., 1991) & \\
\hline $\mathrm{CO}_{3}{ }^{--}+\mathrm{SO}_{3}{ }^{2-} \rightarrow \mathrm{CO}_{3}^{2-}+\mathrm{SO}_{3}^{--}$ & $R(88)$ & $2.910^{7}$ & 470 & (Huie et al., 1991) & \\
\hline $\mathrm{CO}_{3}^{-{ }^{-}}+\mathrm{O}_{3} \rightarrow \mathrm{CO}_{2}+\mathrm{O}_{2}^{--}+\mathrm{O}_{2}$ & $R(89)$ & $1.010^{5}$ & & (Sehested et al., 1983) & \\
\hline $\mathrm{CO}_{3}^{\cdot-}+\mathrm{NO}_{2} \rightarrow \mathrm{CO}_{2}+\mathrm{NO}_{3}^{-}$ & $R(90)$ & $1.010^{9}$ & & (Lilie et al., 1978) & \\
\hline \multicolumn{6}{|l|}{$\mathrm{N}$ chemistry } \\
\hline $\mathrm{HNO}_{2}+\mathrm{hv} \rightarrow \mathrm{NO}+\mathrm{HO}^{\circ}$ & $R(91)$ & Calculated & & $\begin{array}{l}\text { (Graedel and } \\
\text { Weschler, 1981) }\end{array}$ & \\
\hline $\mathrm{NO}_{2}^{-}+\mathrm{hv}+\mathrm{H}_{2} \mathrm{O} \rightarrow \mathrm{NO}+\mathrm{HO}^{\circ}+\mathrm{OH}^{-}$ & $R(92)$ & Calculated & & $\begin{array}{l}\text { (Graedel and } \\
\text { Weschler, 1981); } \\
\text { (Zellner et al., 1990) }\end{array}$ & \\
\hline $\mathrm{HNO}_{2}+\mathrm{H}_{2} \mathrm{O}_{2}+\mathrm{H}^{+} \rightarrow \mathrm{NO}_{3}^{-}+2 \mathrm{H}^{+}+\mathrm{H}_{2} \mathrm{O}$ & $R(93)$ & $6.310^{3}$ & 6700 & (Lee and Lind, 1986) & \\
\hline $\mathrm{NO}_{2}^{-}+\mathrm{O}_{3} \rightarrow \mathrm{NO}_{3}^{-}+\mathrm{O}_{2}$ & $R(94)$ & $5.010^{5}$ & 6900 & $\begin{array}{l}\text { (Damschen and } \\
\text { Martin, 1983) }\end{array}$ & \\
\hline $\mathrm{HNO}_{2}+\mathrm{NO}_{3}{ }^{-} \rightarrow \mathrm{NO}_{2}+\mathrm{NO}_{3}^{-}+\mathrm{H}^{+}$ & $R(95)$ & $8.010^{6}$ & & (Katsumura, 1998) & \\
\hline $\mathrm{NO}_{2}^{-}+\mathrm{NO}_{3}^{-} \rightarrow \mathrm{NO}_{2}+\mathrm{NO}_{3}^{-}$ & $R(96)$ & $1.410^{9}$ & & $\begin{array}{l}\text { (Herrmann and } \\
\text { Zellner, 1998) }\end{array}$ & \\
\hline $\mathrm{NO}_{2}^{-}+\mathrm{CO}_{3}{ }^{-} \rightarrow \mathrm{NO}_{2}+\mathrm{CO}_{3}^{2-}$ & $R(97)$ & $6.610^{5}$ & 850 & (Huie et al., 1991) & \\
\hline $\mathrm{NO}_{2}^{-}+\mathrm{Cl}^{\circ} \rightarrow \mathrm{NO}_{2}+\mathrm{Cl}^{-}$ & $R(98)$ & $5.010^{9}$ & & (Buxton et al., 2000) & \\
\hline $\mathrm{NO}_{2}+\mathrm{HO}_{2}^{\cdot} \rightarrow \mathrm{HNO}_{4}$ & $R(99)$ & $1.810^{9}$ & & $\begin{array}{l}\text { (Logager and } \\
\text { Sehested, 1993) }\end{array}$ & \\
\hline $\mathrm{NO}_{2}+\mathrm{O}_{2}^{-{ }^{-} \rightarrow \mathrm{NO}_{4}^{-}}$ & $R(100)$ & $4.510^{9}$ & & $\begin{array}{l}\text { (Logager and } \\
\text { Sehested, 1993) }\end{array}$ & \\
\hline $\mathrm{HNO}_{4} \rightarrow \mathrm{HO}_{2}^{-}+\mathrm{NO}_{2}$ & $R(101)$ & $2.610^{-2}$ & & $\begin{array}{l}\text { (Goldstein and } \\
\text { Czapski, 1997) }\end{array}$ & \\
\hline $\mathrm{HNO}_{4} \rightarrow \mathrm{HNO}_{2}+\mathrm{O}_{2}$ & $R(102)$ & $7.010^{-4}$ & & $\begin{array}{l}\text { (Logager and } \\
\text { Sehested, 1993) }\end{array}$ & \\
\hline $\mathrm{NO}_{4}^{-} \rightarrow \mathrm{NO}_{2}^{-}+\mathrm{O}_{2}$ & $R(103)$ & 1.1 & & $\begin{array}{l}\text { (Goldstein and } \\
\text { Czapski, 1997) }\end{array}$ & \\
\hline $\mathrm{NO}_{4}^{-} \rightarrow \mathrm{NO}_{2}+\mathrm{O}_{2}^{-\cdot}$ & $R(104)$ & 1.3 & & $\begin{array}{l}\text { (Goldstein and } \\
\text { Czapski, 1997) }\end{array}$ & \\
\hline $\mathrm{HNO}_{4}+\mathrm{HSO}_{3}^{-} \rightarrow \mathrm{SO}_{4}^{2-}+\mathrm{NO}_{3}^{-}+2 \mathrm{H}^{+}$ & $R(105)$ & $3.310^{5}$ & & (Amels et al., 1996) & \\
\hline $\mathrm{NO}_{2}+\mathrm{NO}_{2} \rightarrow \mathrm{HNO}_{2}+\mathrm{NO}_{3}^{-}+\mathrm{H}^{+}-\mathrm{H}_{2} \mathrm{O}$ & $R(106)$ & $8.410^{7}$ & -2900 & (Park and Lee, 1988) & \\
\hline $\mathrm{NO}_{2}+\mathrm{NO} \rightarrow 2 \mathrm{NO}_{2}^{-}+2 \mathrm{H}^{+}-\mathrm{H}_{2} \mathrm{O}$ & $R(107)$ & $3.010^{8}$ & & $\begin{array}{l}\text { (Hoffmann and } \\
\text { Calvert, 1985) }\end{array}$ & \\
\hline $\mathrm{NO}_{3}^{-}+\mathrm{hv} \rightarrow \mathrm{NO}_{2}+\mathrm{HO}^{\circ}+\mathrm{OH}^{-}-\mathrm{H}_{2} \mathrm{O}$ & $R(108)$ & Calculated & & $\begin{array}{l}\text { (Graedel and } \\
\text { Weschler, 1981); } \\
\text { (Zellner et al., 1990) }\end{array}$ & \\
\hline $\mathrm{N}_{2} \mathrm{O}_{5} \rightarrow 2 \mathrm{HNO}_{3}-\mathrm{H}_{2} \mathrm{O}$ & $R(109)$ & $1.010^{6}$ & & Estimated & 5 \\
\hline $\mathrm{NO}_{3}^{\circ}+\mathrm{hv} \rightarrow \mathrm{NO}+\mathrm{O}_{2}$ & $R(110)$ & Calculated & & (Graedel and & \\
\hline
\end{tabular}




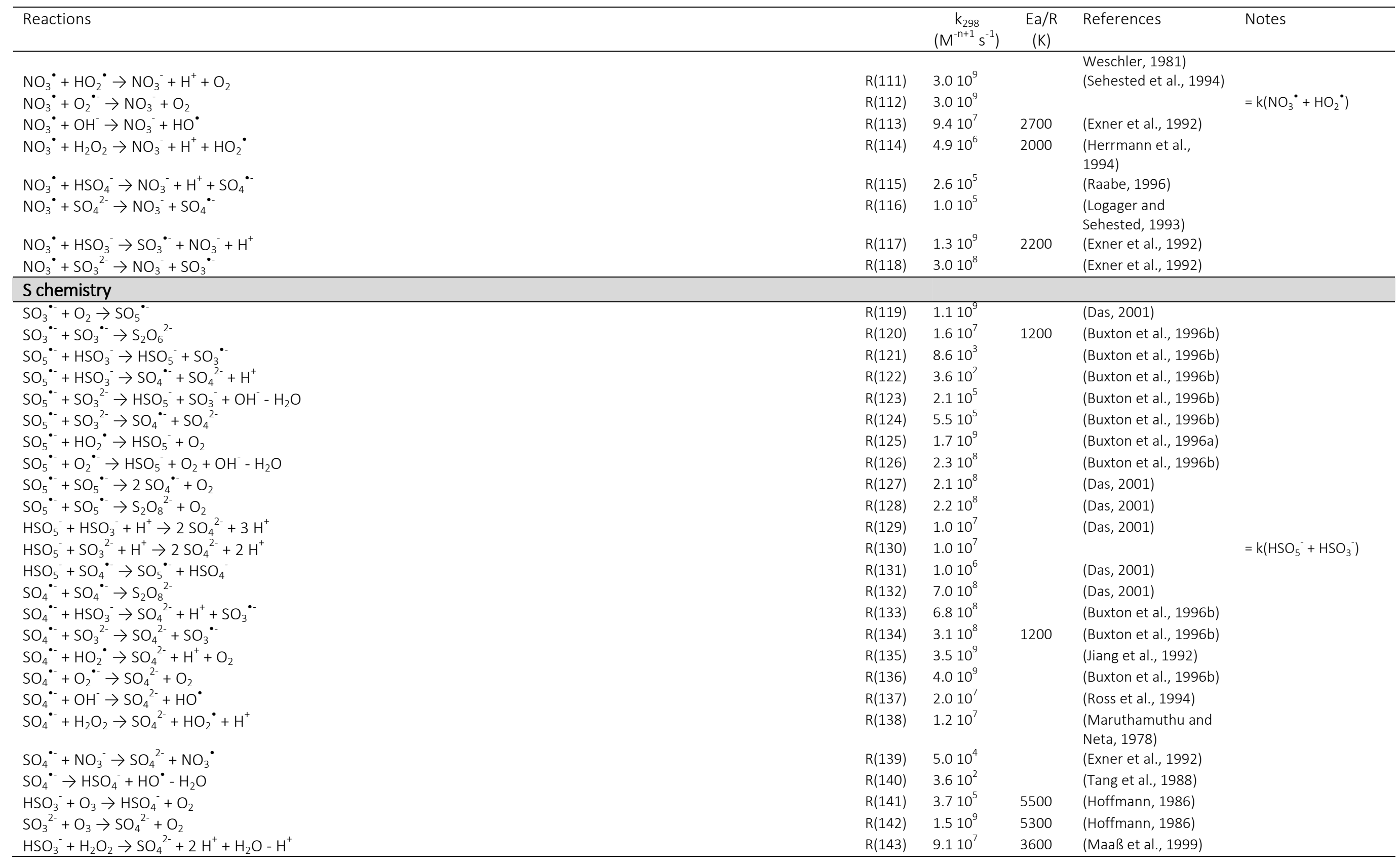


1 - Sehested et al. (1984) argue that this reaction is very slow with a rate constant lower than $10^{4} \mathrm{M}^{-1} \mathrm{~s}^{-1}$.

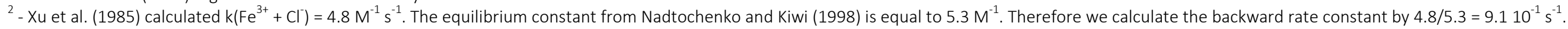

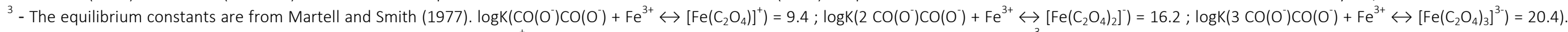

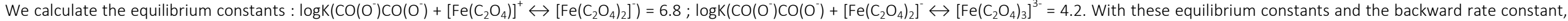
we calculate the forward rate constants.

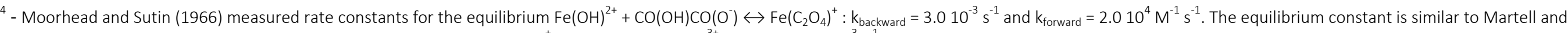
Smith (1977). We suppose that the rate constant of the reaction $\left[\mathrm{Fe}\left(\mathrm{C}_{2} \mathrm{O}_{4}\right)\right]^{+} \rightarrow \mathrm{CO}\left(\mathrm{O}^{-}\right) \mathrm{CO}\left(\mathrm{O}^{-}\right)+\mathrm{Fe}^{3+}$ is equal to $3.010^{-3} \mathrm{~s}^{-1}$

${ }^{5}$ - We suppose that the $\mathrm{N}_{2} \mathrm{O}_{5}$ hydrolysis is fast with a first order rate constant equal to $10^{6} \mathrm{~s}^{-1}$ (Bertram and Thornton, 2009). 
Equilibria

\begin{tabular}{|c|c|c|c|c|c|}
\hline Species & & $\mathrm{K}_{\mathrm{a}}$ or $\mathrm{K}_{\mathrm{h}}$ & $\begin{array}{c}-\Delta H / R \\
(K)\end{array}$ & References & Notes \\
\hline $\mathrm{H}_{2} \mathrm{O}_{2} \leftrightarrow \mathrm{HO}_{2}^{-}+\mathrm{H}^{+}$ & $T(1)$ & $2.210^{-12}$ & -3730 & $\begin{array}{l}\text { (Smith and Martell, } \\
\text { 1976) }\end{array}$ & \\
\hline $\mathrm{HO}_{2}{ }^{\bullet} \leftrightarrow \mathrm{O}_{2}{ }^{\cdot-}+\mathrm{H}^{+}$ & $T(2)$ & $1.610^{-5}$ & & (Bielski et al., 1985) & \\
\hline $\mathrm{HNO}_{2} \leftrightarrow \mathrm{NO}_{2}^{-}+\mathrm{H}^{+}$ & $T(3)$ & $1.610^{-3}$ & 1760 & $\begin{array}{l}\text { (Park and Lee, 1988); } \\
\text { (Riordan et al., 2005) }\end{array}$ & \\
\hline $\mathrm{HNO}_{3} \leftrightarrow \mathrm{NO}_{3}^{-}+\mathrm{H}^{+}$ & $\mathrm{T}(4)$ & $2.210^{1}$ & & (N.B.S, 1965) & \\
\hline $\mathrm{HNO}_{4} \leftrightarrow \mathrm{NO}_{4}^{-}+\mathrm{H}^{+}$ & $T(5)$ & $1.310^{-6}$ & & $\begin{array}{l}\text { (Goldstein and Czapski, } \\
\text { 1997) }\end{array}$ & \\
\hline $\mathrm{HCl} \leftrightarrow \mathrm{Cl}^{-}+\mathrm{H}^{+}$ & $T(6)$ & $1.710^{-6}$ & -6890 & $\begin{array}{l}\text { (Marsh and McElroy, } \\
\text { 1985) }\end{array}$ & \\
\hline $\mathrm{SO}_{2}+\mathrm{H}_{2} \mathrm{O} \leftrightarrow \mathrm{HSO}_{3}^{-}+\mathrm{H}^{+}$ & $T(7)$ & $1.310^{-2}$ & -1960 & (Maash, 1982) & \\
\hline $\mathrm{HSO}_{3}^{-} \leftrightarrow \mathrm{SO}_{3}^{2-}+\mathrm{H}^{+}$ & $\mathrm{T}(8)$ & $6.410^{-8}$ & -1430 & (Maash, 1982) & \\
\hline $\mathrm{H}_{2} \mathrm{SO}_{4} \leftrightarrow \mathrm{HSO}_{4}^{-}+\mathrm{H}^{+}$ & $\mathrm{T}(9)$ & $1.010^{3}$ & & $\begin{array}{l}\text { (Cotton and Wilkinson, } \\
\text { 1980) }\end{array}$ & \\
\hline $\mathrm{HSO}_{4}^{-} \leftrightarrow \mathrm{SO}_{4}^{2-}+\mathrm{H}^{+}$ & $T(10)$ & $1.010^{-2}$ & & (Eigen et al., 1964) & \\
\hline $\mathrm{Fe}^{3+}+\mathrm{H}_{2} \mathrm{O} \leftrightarrow[\mathrm{Fe}(\mathrm{OH})]^{2+}+\mathrm{H}^{+}$ & $T(11)$ & $6.010^{-3}$ & & $\begin{array}{l}\text { (Brandt and van Eldik, } \\
\text { 1995) }\end{array}$ & \\
\hline$[\mathrm{Fe}(\mathrm{OH})]^{2+}+\mathrm{H}_{2} \mathrm{O} \leftrightarrow\left[\mathrm{Fe}(\mathrm{OH})_{2}\right]^{+}+\mathrm{H}^{+}$ & $T(12)$ & $7.610^{-4}$ & & $\begin{array}{l}\text { (Brandt and van Eldik, } \\
\text { 1995) }\end{array}$ & \\
\hline $\mathrm{CO}_{2}+\mathrm{H}_{2} \mathrm{O} \leftrightarrow \mathrm{HCO}_{3}^{-}+\mathrm{H}^{+}$ & $T(13)$ & $4.210^{-7}$ & & $\begin{array}{l}\text { (Cotton and Wilkinson, } \\
\text { 1980) }\end{array}$ & \\
\hline $\mathrm{HCO}_{3}{ }^{-} \leftrightarrow \mathrm{CO}_{3}{ }^{2-}+\mathrm{H}^{+}$ & $T(14)$ & $4.810^{-11}$ & & $\begin{array}{l}\text { (Cotton and Wilkinson, } \\
\text { 1980) }\end{array}$ & \\
\hline
\end{tabular}

Table SM1: Chemical mechanism considered in the M2C2 model. 


\begin{tabular}{|c|c|c|c|c|c|c|c|c|c|c|c|c|c|c|c|c|c|c|c|}
\hline \multirow{2}{*}{ Date } & & \multirow{2}{*}{ Origin } & \multirow{2}{*}{$\begin{array}{c}\text { Temperature } \\
\left({ }^{\circ} \mathrm{C}\right)\end{array}$} & \multirow{2}{*}{ pH } & \multirow{2}{*}{$\begin{array}{c}\text { Redox } \\
\text { potential } \\
(\mathbf{m V})\end{array}$} & \multirow{2}{*}{$\begin{array}{c}\text { TOC } \\
(\mathrm{mg} / \mathrm{L})\end{array}$} & \multirow{2}{*}{$\begin{array}{c}\text { TC } \\
(\mathrm{mg} / \mathrm{L})\end{array}$} & \multirow{2}{*}{$\begin{array}{c}\mathrm{IC} \\
(\mathrm{mg} / \mathrm{L})\end{array}$} & \multicolumn{6}{|c|}{ Anions $(\mu \mathrm{mol} / \mathrm{L})$} & \multicolumn{5}{|c|}{ Cations $(\mu \mathrm{mol} / \mathrm{L})$} \\
\hline & & & & & & & & & $\mathrm{SO}_{4}{ }^{2-}$ & $\mathrm{Cl}^{-}$ & Acetate & Formate & Oxalate & Succinate & $\mathbf{N a}^{+}$ & $\mathrm{NH}_{4}^{+}$ & $\mathrm{Mg}^{2+}$ & $\mathbf{K}^{+}$ & $\mathrm{Ca}^{2+}$ \\
\hline $10 / 14 / 2013$ & 4PM-6PM & W & 6 & 6.1 & NM & $\mathrm{NM}$ & NM & 2.7 & 23.0 & 45.9 & 8.7 & 34.8 & 4.5 & 3.3 & 18.5 & 66.2 & 33.7 & 7.2 & 2.4 \\
\hline $10 / 14 / 2013$ & 6PM-9PM & $\mathrm{W}$ & 6.5 & 5.6 & $\mathrm{NM}$ & $\mathrm{NM}$ & $\mathrm{NM}$ & 0.9 & 16.7 & 82.5 & 3.3 & 20.4 & 2.5 & 3.7 & 7.6 & 33.0 & 6.4 & 33.1 & 0.4 \\
\hline $10 / 14 / 2013$ & 9PM-12PM & $\mathrm{W}$ & 6.7 & 5.6 & NM & 1.6 & 2.2 & 0.7 & 18.5 & 46.2 & 2.6 & 11.7 & 1.8 & 4.2 & 11.9 & 44.6 & 5.2 & 20.9 & 3.8 \\
\hline $10 / 29 / 2013$ & 5АM-8АM & $\mathrm{W}$ & 2.2 & 5.0 & 261 & 2.8 & 3.6 & 0.8 & 46.8 & 246.9 & 21.1 & 28.5 & 2.7 & 4.2 & 56.3 & 38.0 & $\mathrm{NM}$ & 4.8 & NM \\
\hline $10 / 29 / 2013$ & $8 \mathrm{AM}-12 \mathrm{AM}$ & $\mathrm{W}$ & 3.1 & 5.6 & 248 & 3.2 & 3.3 & 0.1 & 55.8 & 269.5 & 19.1 & 25.3 & 2.8 & 5.2 & 76.3 & 64.0 & $\mathrm{NM}$ & 11.7 & NM \\
\hline $11 / 5 / 2013$ & 2PM-4PM & W & 6 & 5.8 & 231 & 2.0 & 2.7 & 0.7 & 8.5 & 25.0 & 13.9 & 25.5 & 1.9 & 3.4 & 7.8 & 13.5 & NM & 34.1 & NM \\
\hline $11 / 5 / 2013$ & 4PM-7PM & $\mathrm{W}$ & 6 & 5.5 & 263 & 1.1 & 1.7 & 0.6 & 4.5 & 7.2 & 17.5 & 12.4 & 1.3 & 3.9 & 3.7 & 8.9 & NM & 25.7 & NM \\
\hline $11 / 5 / 2013$ & 7PM-9PM & $\mathrm{W}$ & 5.8 & 5.6 & 313 & 0.8 & 1.4 & 0.56 & 7.9 & 16.6 & 9.3 & 24.5 & 1.7 & 3.0 & 4.5 & 17.7 & $\mathrm{NM}$ & 21.9 & NM \\
\hline $11 / 5 / 2013$ & 9PM-12PM & $\mathrm{W}$ & 5.5 & 5.0 & 290 & 0.6 & 1.5 & 0.8 & 9.8 & 16.9 & 5.7 & 7.2 & 1.3 & 2.3 & 6.5 & 26.7 & $\mathrm{NM}$ & 22.7 & NM \\
\hline $11 / 6 / 2013$ & 7AM-10AM & W & 7.9 & 5.4 & 289 & 1.1 & 1.9 & 0.9 & 6.1 & 9.7 & 7.5 & 17.2 & 1.3 & 5.7 & 6.0 & 18.0 & $\mathrm{NM}$ & 24.3 & NM \\
\hline $11 / 6 / 2013$ & 10AM-12AM & $\mathrm{W}$ & 8 & 5.7 & 352 & 1.9 & 2.6 & 0.7 & 6.7 & 5.8 & 7.5 & 9.5 & 1.5 & 2.7 & 3.3 & 27.0 & $\mathrm{NM}$ & 25.8 & NM \\
\hline $3 / 22 / 2014$ & 7AM-11AM & W & 0 & 6.7 & 228 & 2.9 & 3.5 & 0.6 & 34.8 & 65.7 & 13.3 & 48.3 & 4.1 & 0.1 & 20.9 & 87.6 & 12.4 & 2.3 & 4.7 \\
\hline $3 / 22 / 2014$ & $11 \mathrm{AM}-2 \mathrm{PM}$ & W & 0 & 6.7 & 264 & 5.4 & 6.1 & 0.7 & 37.1 & 133.8 & 20.4 & 43.8 & 5.4 & 0.2 & 36.8 & 108.8 & 8.7 & 4.9 & 6.6 \\
\hline $3 / 25 / 2014$ & $11 \mathrm{AM}-1 \mathrm{PM}$ & W & -2 & 6.6 & 240 & 3.4 & 3.9 & 0.5 & 49.2 & 228.5 & 13.8 & 30.6 & 3.2 & 0.1 & 56.8 & 73.2 & 10.3 & 9.1 & 7.1 \\
\hline $3 / 25 / 2014$ & 1PM-3PM & NW & 0 & 6.4 & 228 & 6.0 & 6.6 & 0.5 & 32.6 & 153.8 & 14.0 & 65.1 & 3.3 & 0.2 & 38.8 & 79.6 & 12.5 & 6.4 & 6.9 \\
\hline $3 / 25 / 2014$ & 7PM-9PM & NW & -2 & 6.1 & 243 & 8.7 & 9.0 & 0.4 & 14.8 & 73.0 & 12.5 & 65.0 & 3.8 & 0.2 & 20.8 & 50.2 & 13.6 & 4.0 & 9.4 \\
\hline $3 / 26 / 2014$ & 8AM-9AM & $\mathrm{N}$ & -3 & 5.5 & 233 & 6.5 & 7.0 & 0.5 & 56.4 & 25.2 & 26.2 & 38.3 & 5.9 & 0.8 & 9.6 & 252.4 & 7.2 & 2.9 & 7.9 \\
\hline $3 / 26 / 2014$ & $9 \mathrm{AM}-11 \mathrm{AM}$ & $\mathrm{N}$ & -3 & 5.4 & 239 & 8.6 & 9.2 & 0.6 & 45.4 & 26.5 & 15.8 & 34.9 & 5.3 & 0.7 & 8.3 & 206.0 & 9.0 & 2.4 & 15.6 \\
\hline $4 / 4 / 2014$ & 8PM-10PM & NW & 2 & 6.2 & 261 & 1.5 & 2.0 & 0.5 & 13.5 & 19.4 & 9.6 & 23.6 & 3.4 & 0.4 & 5.0 & 39.6 & 5.4 & 7.8 & 0.0 \\
\hline $4 / 4 / 2014$ & 10PM-12PM & $\mathrm{NW}$ & 2 & 6.5 & 261 & 1.7 & 2.1 & 0.4 & 18.2 & 18.2 & 7.2 & 24.2 & 3.8 & 0.3 & 3.9 & 71.4 & 4.3 & 7.1 & 0.0 \\
\hline $4 / 5 / 2014$ & 1PM-4AM & W & 2 & 6.6 & 250 & 2.6 & 3.1 & 0.5 & 26.0 & 29.4 & 14.3 & 32.5 & 4.4 & 0.3 & 6.9 & 124.1 & 4.4 & 7.4 & 0.0 \\
\hline $4 / 5 / 2014$ & 4AM-7AM & W & 2 & 6.9 & 237 & 3.9 & 4.6 & 0.7 & 39.8 & 48.7 & 25.4 & 48.8 & 6.9 & 0.4 & 12.6 & 192.8 & 4.3 & 4.8 & 0.0 \\
\hline $4 / 5 / 2014$ & 7AM-10AM & W & 2 & 6.8 & 239 & 4.5 & 5.3 & 0.7 & 67.5 & 76.1 & 30.4 & 63.4 & 7.4 & 0.5 & 18.0 & 212.7 & 5.3 & 4.3 & 2.0 \\
\hline $4 / 11 / 2014$ & $11 \mathrm{AM}-1 \mathrm{PM}$ & $\mathrm{W}$ & 1 & 5.4 & 207 & 1.9 & 1.9 & 0.0 & 7.5 & 14.0 & 7.6 & 3.6 & 5.4 & 6.2 & 27.7 & $\mathrm{NM}$ & $\mathrm{NM}$ & 31.9 & NM \\
\hline $5 / 11 / 2014$ & 4AM-6PM & $\mathrm{W}$ & 0.3 & 4.7 & 238 & 1.8 & 1.8 & 0.0 & 58.1 & 35.4 & 11.8 & 3.8 & 113.2 & 13.9 & 51.4 & $\mathrm{NM}$ & $\mathrm{NM}$ & 26.0 & NM \\
\hline $5 / 11 / 2014$ & 7АM-9AM & NW & 0 & 4.1 & 280 & 1.8 & 1.8 & 0.0 & 139.3 & 41.7 & 5.2 & 3.3 & 115.7 & 9.2 & 43.8 & 39.7 & NM & 20.3 & NM \\
\hline
\end{tabular}




\begin{tabular}{|c|c|c|c|c|c|c|c|c|c|c|c|c|c|c|c|c|c|c|c|c|}
\hline 27 & $5 / 11 / 2014$ & 9AM-12AM & NW & -0.1 & 4.1 & 298 & $\mathrm{NM}$ & 14.9 & 0.1 & 247.4 & 98.0 & 3.8 & 3.4 & 302.2 & 18.8 & 99.9 & 83.9 & $\mathrm{NM}$ & 17.8 & NM \\
\hline 28 & $5 / 11 / 2014$ & 12AM-1PM & NW & 0.1 & 4.2 & 295 & 3.6 & 3.6 & 0.0 & 202.8 & 87.0 & 10.8 & 3.1 & 377.2 & 27.9 & 116.7 & 97.37 & $\mathrm{NM}$ & 21.7 & NM \\
\hline 29 & $12 / 11 / 2014$ & 3РM-5PM & $\mathrm{W}$ & 3 & 5.4 & 250 & 4.0 & 4.0 & 0.0 & 38.3 & 78.9 & 11.9 & 5.4 & 83.2 & 21.9 & 83.5 & 15.1 & NM & 19.9 & NM \\
\hline 30 & $12 / 11 / 2014$ & 5PM-7PM & $\mathrm{W}$ & 2 & 5.5 & 261 & 2.3 & 2.4 & 0.1 & 28.7 & 68.2 & 12.7 & 4.8 & 47.1 & 21.7 & 72.2 & $\mathrm{NM}$ & $\mathrm{NM}$ & 17.6 & NM \\
\hline 31 & $12 / 11 / 2014$ & 7PM-9PM & $\mathrm{W}$ & 2 & 5.6 & 254 & 2.7 & 2.8 & 0.1 & 24.2 & 68.9 & 2.9 & 2.9 & 32.5 & 33.0 & 77.3 & $\mathrm{NM}$ & $\mathrm{NM}$ & 21.3 & NM \\
\hline 32 & $12 / 11 / 2014$ & 9PM-12PM & $\mathrm{W}$ & 2 & 5.4 & 254 & 1.7 & 1.7 & 0.1 & 23.4 & 64.4 & 5.2 & 4.5 & 20.2 & 13.5 & 80.1 & $\mathrm{NM}$ & $\mathrm{NM}$ & 17.5 & NM \\
\hline 33 & $14 / 11 / 2014$ & 5PM-7PM & $S$ & 5 & 5.5 & 258 & 2.2 & 2.2 & 0.0 & 11.3 & 6.0 & 4.9 & 3.6 & 10.4 & 5.4 & 15.7 & $\mathrm{NM}$ & $\mathrm{NM}$ & 16.3 & NM \\
\hline 34 & $17 / 11 / 2014$ & 4PM-6РM & $\mathrm{W}$ & 1.1 & 5.6 & 249 & 2.2 & 2.4 & 0.1 & 8.8 & 4.7 & 7.6 & 3.9 & 11.8 & 5.6 & 13.1 & $\mathrm{NM}$ & $\mathrm{NM}$ & 21.3 & NM \\
\hline 35 & $17 / 11 / 2014$ & 7PM-9PM & $\mathrm{W}$ & 0.7 & 5.7 & 245 & 1.0 & 1.1 & 0.0 & 8.0 & 2.0 & 7.3 & 3.6 & 10.0 & 5.8 & 10.9 & $\mathrm{NM}$ & $\mathrm{NM}$ & 19.9 & NM \\
\hline 36 & $17 / 11 / 2014$ & 9PM-12PM & $\mathrm{W}$ & 0.6 & 5.3 & 263 & 1.2 & 1.2 & 0.0 & 11.0 & 5.3 & 6.9 & 3.8 & 14.3 & 6.9 & 14.2 & $\mathrm{NM}$ & $\mathrm{NM}$ & 18.5 & NM \\
\hline 37 & $18 / 11 / 2014$ & 6AM-8AM & NW & 0.1 & 5.4 & 258 & 1.0 & 0.9 & 0.0 & 28.2 & 16.5 & 4.2 & 3.4 & 32.6 & 7.2 & 23.0 & $\mathrm{NM}$ & $\mathrm{NM}$ & 19.1 & NM \\
\hline 38 & $18 / 11 / 2014$ & 5PM-7PM & NW & 1.8 & 5.7 & 243 & 2.3 & 2.4 & 0.1 & 16.4 & 11.9 & 8.8 & 3.6 & 22.0 & 7.0 & 18.3 & 2.2 & $\mathrm{NM}$ & 20.2 & NM \\
\hline 39 & $18 / 11 / 2014$ & 7PM-9PM & NW & 1 & 5.3 & 257 & 1.8 & 1.8 & 0.0 & 14.0 & 7.3 & 5.6 & 3.7 & 14.1 & 5.6 & 18.2 & NM & NM & 20.2 & NM \\
\hline 40 & $18 / 11 / 2014$ & 9PM-12PM & NW & 1 & 4.9 & 284 & 1.9 & 1.9 & 0.1 & 20.0 & 18.0 & 7.0 & 3.6 & 16.0 & 8.3 & 17.7 & $\mathrm{NM}$ & NM & 18.7 & NM \\
\hline 41 & $19 / 11 / 2014$ & 12PM-2AM & NW & 1 & 4.6 & 306 & 2.2 & 2.2 & 0.0 & 26.7 & 36.7 & 10.3 & 3.4 & 34.7 & 12.3 & 28.1 & NM & NM & 17.1 & NM \\
\hline
\end{tabular}

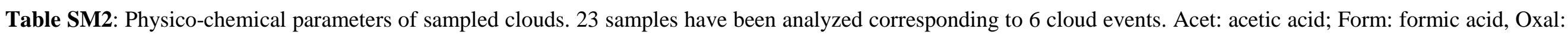
oxalic acid, Succ: succinic acid). NM: not measured. 


\begin{tabular}{|c|c|c|c|c|c|c|c|}
\hline \multirow{2}{*}{ Cloud sample } & \multicolumn{5}{|c|}{ Relative contribution $(\%)$} & \multirow{2}{*}{$\mathrm{R}_{\mathrm{HO}}^{\mathrm{f}} \cdot \bmod \left(\mathrm{M} \mathrm{s}^{-1}\right)$} & \multirow{2}{*}{$\mathrm{R}_{\mathrm{HO}}^{\mathrm{f}} \cdot \exp \left(\mathrm{M} \mathrm{s}^{-1}\right)$} \\
\hline & $\mathrm{H}_{2} \mathrm{O}_{2}+\mathrm{h} v$ & $\mathrm{Fe}(\mathrm{II})+\mathrm{H}_{2} \mathrm{O}_{2}$ & $\mathrm{Fe}(\mathrm{III})+\mathrm{h} v$ & $\mathrm{NO}_{3}^{-}+\mathrm{hv}$ & $\mathrm{HNO}_{2}+\mathrm{NO}_{2}^{-}+\mathrm{hv}$ & & \\
\hline 1 & 86.7 & 0.0 & 0.0 & 2.6 & 10.6 & $4.3 \times 10^{-11}$ & $3.3 \times 10^{-11}$ \\
\hline 2 & 64.9 & 0.0 & 0.0 & 1.0 & 34.1 & $4.2 \times 10^{-11}$ & NM \\
\hline 3 & 90.7 & 0.0 & 0.0 & 1.3 & 7.9 & $5.0 \times 10^{-11}$ & NM \\
\hline 4 & 91.2 & 0.0 & 0.0 & 2.0 & 6.7 & $4.6 \times 10^{-11}$ & $1.4 \times 10^{-10}$ \\
\hline 5 & 89.1 & 0.0 & 0.0 & 2.2 & 8.6 & $4.4 \times 10^{-11}$ & $1.2 \times 10^{-10}$ \\
\hline 6 & 99.3 & 0.0 & 0.0 & 0.7 & 0.0 & $2.4 \times 10^{-11}$ & $2.8 \times 10^{-11}$ \\
\hline 7 & 99.4 & 0.0 & 0.0 & 0.6 & 0.0 & $1.9 \times 10^{-11}$ & $5.6 \times 10^{-11}$ \\
\hline 8 & 98.4 & 0.0 & 0.0 & 1.6 & 0.0 & $3.0 \times 10^{-11}$ & $2.5 \times 10^{-11}$ \\
\hline 9 & 97.8 & 0.0 & 0.0 & 2.2 & 0.0 & $2.5 \times 10^{-11}$ & $2.2 \times 10^{-11}$ \\
\hline 10 & 99.5 & 0.0 & 0.0 & 0.5 & 0.0 & $3.1 \times 10^{-11}$ & $2.9 \times 10^{-11}$ \\
\hline 11 & 99.3 & 0.0 & 0.0 & 0.7 & 0.0 & $5.2 \times 10^{-11}$ & $6.8 \times 10^{-11}$ \\
\hline 12 & 22.3 & 21.6 & 54.2 & 0.7 & 1.2 & $2.4 \times 10^{-10}$ & $6.1 \times 10^{-11}$ \\
\hline 13 & 57.3 & 10.7 & 22.4 & 1.2 & 8.4 & $1.3 \times 10^{-10}$ & $4.7 \times 10^{-11}$ \\
\hline 14 & 52.4 & 10.7 & 35.1 & 1.8 & 0.0 & $6.9 \times 10^{-11}$ & $2.8 \times 10^{-11}$ \\
\hline 15 & 92.5 & 0.0 & 0.0 & 2.7 & 4.9 & $4.7 \times 10^{-11}$ & $1.1 \times 10^{-11}$ \\
\hline 16 & 91.8 & 0.0 & 0.0 & 4.8 & 3.4 & $3.0 \times 10^{-11}$ & NM \\
\hline 17 & 50.2 & 12.4 & 21.7 & 15.1 & 0.7 & $9.8 \times 10^{-11}$ & $6.1 \times 10^{-11}$ \\
\hline 18 & 51.8 & 22.3 & 10.4 & 14.6 & 0.9 & $9.4 \times 10^{-11}$ & $3.4 \times 10^{-11}$ \\
\hline 19 & 94.6 & 0.0 & 0.0 & 2.8 & 2.6 & $4.8 \times 10^{-11}$ & $8.1 \times 10^{-11}$ \\
\hline 20 & 91.4 & 0.0 & 0.0 & 4.8 & 3.7 & $5.2 \times 10^{-11}$ & $8.5 \times 10^{-11}$ \\
\hline 21 & 88.0 & 0.0 & 0.0 & 6.4 & 5.6 & $7.6 \times 10^{-11}$ & $1.5 \times 10^{-10}$ \\
\hline 22 & 90.7 & 0.0 & 0.0 & 5.1 & 4.2 & $1.7 \times 10^{-10}$ & $3.4 \times 10^{-10}$ \\
\hline 23 & 89.0 & 0.0 & 0.0 & 5.3 & 5.7 & $1.7 \times 10^{-10}$ & $4.2 \times 10^{-10}$ \\
\hline 24 & 98.0 & 0.0 & 0.0 & 2.0 & 0.0 & $2.5 \times 10^{-11}$ & $5.1 \times 10^{-11}$ \\
\hline 25 & 60.6 & 29.2 & 1.1 & 4.2 & 4.9 & $3.4 \times 10^{-11}$ & $2.4 \times 10^{-11}$ \\
\hline 26 & 26.2 & 70.0 & 1.1 & 2.6 & 0.1 & $1.0 \times 10^{-10}$ & $1.4 \times 10^{-10}$ \\
\hline 27 & 19.3 & 76.8 & 0.9 & 3.0 & 0.1 & $1.8 \times 10^{-10}$ & $5.0 \times 10^{-11}$ \\
\hline 28 & 17.6 & 79.0 & 1.0 & 2.4 & 0.0 & $2.2 \times 10^{-10}$ & NM \\
\hline
\end{tabular}




\begin{tabular}{|c|c|c|c|c|c|c|c|}
\hline 29 & 39.8 & 40.3 & 12.7 & 2.7 & 4.5 & $6.2 \times 10^{-11}$ & $8.5 \times 10^{-11}$ \\
\hline 30 & 43.4 & 27.3 & 18.6 & 2.2 & 8.5 & $6.2 \times 10^{-11}$ & $8.4 \times 10^{-11}$ \\
\hline 31 & 21.7 & 33.0 & 39.4 & 1.1 & 4.8 & $1.3 \times 10^{-10}$ & $6.1 \times 10^{-11}$ \\
\hline 32 & 92.1 & 1.6 & 1.3 & 3.3 & 1.7 & $4.3 \times 10^{-11}$ & NM \\
\hline 33 & 31.0 & 10.2 & 56.8 & 2.0 & 0.0 & $2.3 \times 10^{-11}$ & $3.3 \times 10^{-12}$ \\
\hline 34 & 16.6 & 27.9 & 52.0 & 0.5 & 3.1 & $1.5 \times 10^{-10}$ & $2.7 \times 10^{-11}$ \\
\hline 35 & 24.0 & 5.5 & 48.5 & 3.7 & 18.4 & $2.9 \times 10^{-11}$ & $3.6 \times 10^{-11}$ \\
\hline 36 & 60.7 & 7.3 & 18.9 & 13.0 & 0.2 & $1.1 \times 10^{-11}$ & $6.0 \times 10^{-11}$ \\
\hline 37 & 63.5 & 8.3 & 15.6 & 12.4 & 0.2 & $1.0 \times 10^{-11}$ & $2.4 \times 10^{-11}$ \\
\hline 38 & 47.7 & 17.3 & 6.7 & 5.9 & 22.4 & $1.5 \times 10^{-11}$ & $5.8 \times 10^{-11}$ \\
\hline 39 & 67.5 & 14.7 & 7.7 & 10.0 & 0.1 & $1.4 \times 10^{-11}$ & $2.7 \times 10^{-11}$ \\
\hline 40 & 81.6 & 4.5 & 1.3 & 12.4 & 0.2 & $2.1 \times 10^{-11}$ & $1.3 \times 10^{-10}$ \\
\hline 41 & 47.0 & 35.9 & 2.9 & 9.0 & 5.3 & $3.5 \times 10^{-11}$ & $1.1 \times 10^{-10}$ \\
\hline
\end{tabular}

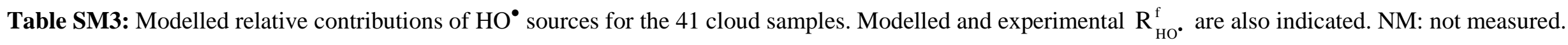




\begin{tabular}{|c|c|c|c|c|c|}
\hline \multirow{2}{*}{ Cloud sample } & \multicolumn{3}{|c|}{ Relative contribution $(\%)$} & \multirow{2}{*}{$R_{H O}^{f} \cdot \bmod \left(\mathrm{M} \mathrm{s}^{-1}\right)$} & \multirow{2}{*}{$R_{H O^{\circ}}^{f} \exp \left(\mathrm{M} \mathrm{s}^{-1}\right)$} \\
\hline & $\mathrm{H}_{2} \mathrm{O}_{2}+\mathrm{hv}$ & $\mathrm{NO}_{3}^{-}+\mathrm{hv}$ & $\mathrm{HNO}_{2}+\mathrm{NO}_{2}^{-}+\mathrm{hv}$ & & \\
\hline 1 & 93.2 & 3.1 & 3.7 & $6.6 \times 10^{-11}$ & $3.3 \times 10^{-11}$ \\
\hline 2 & 84.4 & 1.4 & 14.2 & $5.3 \times 10^{-11}$ & NM \\
\hline 3 & 95.7 & 1.6 & 2.7 & $7.9 \times 10^{-11}$ & NM \\
\hline 4 & 95.3 & 2.4 & 2.4 & $7.3 \times 10^{-11}$ & $1.4 \times 10^{-10}$ \\
\hline 5 & 94.4 & 2.6 & 3.0 & $6.9 \times 10^{-11}$ & $1.2 \times 10^{-10}$ \\
\hline 6 & 99.2 & 0.8 & 0.0 & $3.9 \times 10^{-11}$ & $2.8 \times 10^{-11}$ \\
\hline 7 & 99.3 & 0.7 & 0.0 & $3.1 \times 10^{-11}$ & $5.6 \times 10^{-11}$ \\
\hline 8 & 98.3 & 1.7 & 0.0 & $4.9 \times 10^{-11}$ & $2.5 \times 10^{-11}$ \\
\hline 9 & 97.6 & 2.4 & 0.0 & $4.2 \times 10^{-11}$ & $2.2 \times 10^{-11}$ \\
\hline 10 & 99.4 & 0.6 & 0.0 & $5.1 \times 10^{-11}$ & $2.9 \times 10^{-11}$ \\
\hline 11 & 99.2 & 0.8 & 0.0 & $8.6 \times 10^{-11}$ & $6.8 \times 10^{-11}$ \\
\hline 12 & 95.2 & 3.2 & 1.6 & $9.4 \times 10^{-11}$ & $6.1 \times 10^{-11}$ \\
\hline 13 & 93.4 & 2.2 & 4.3 & $1.3 \times 10^{-10}$ & $4.7 \times 10^{-11}$ \\
\hline 14 & 96.2 & 3.8 & 0.0 & $6.2 \times 10^{-11}$ & $2.8 \times 10^{-11}$ \\
\hline 15 & 95.3 & 3.1 & 1.6 & $7.6 \times 10^{-11}$ & $1.1 \times 10^{-11}$ \\
\hline 16 & 93.5 & 5.4 & 1.1 & $4.8 \times 10^{-11}$ & NM \\
\hline 17 & 74.6 & 25.0 & 0.4 & $1.1 \times 10^{-10}$ & $6.1 \times 10^{-11}$ \\
\hline 18 & 75.8 & 23.8 & 0.5 & $1.1 \times 10^{-10}$ & $3.4 \times 10^{-11}$ \\
\hline 19 & 96.0 & 3.2 & 0.8 & $7.7 \times 10^{-11}$ & $8.1 \times 10^{-11}$ \\
\hline 20 & 93.3 & 5.5 & 1.2 & $8.4 \times 10^{-11}$ & $8.5 \times 10^{-11}$ \\
\hline 21 & 90.8 & 7.3 & 1.8 & $1.2 \times 10^{-10}$ & $1.5 \times 10^{-10}$ \\
\hline 22 & 92.9 & 5.8 & 1.4 & $2.8 \times 10^{-10}$ & $3.4 \times 10^{-10}$ \\
\hline 23 & 92.0 & 6.1 & 1.9 & $2.7 \times 10^{-10}$ & $4.2 \times 10^{-10}$ \\
\hline 24 & 97.7 & 2.2 & 0.0 & $4.1 \times 10^{-11}$ & $5.1 \times 10^{-11}$ \\
\hline 25 & 90.4 & 7.1 & 2.6 & $3.7 \times 10^{-11}$ & $2.4 \times 10^{-11}$ \\
\hline 26 & 87.3 & 12.6 & 0.1 & $3.9 \times 10^{-11}$ & $1.4 \times 10^{-10}$ \\
\hline 27 & 77.8 & 22.0 & 0.2 & $4.2 \times 10^{-11}$ & $5.0 \times 10^{-11}$ \\
\hline 28 & 79.7 & 20.2 & 0.2 & $4.5 \times 10^{-11}$ & $\mathrm{NM}$ \\
\hline 29 & 89.8 & 6.8 & 3.3 & $4.4 \times 10^{-11}$ & $8.5 \times 10^{-11}$ \\
\hline 30 & 89.4 & 5.0 & 5.7 & $4.9 \times 10^{-11}$ & $8.4 \times 10^{-11}$ \\
\hline 31 & 88.7 & 5.0 & 6.3 & $5.1 \times 10^{-11}$ & $6.1 \times 10^{-11}$ \\
\hline 32 & 95.6 & 3.8 & 0.6 & $6.8 \times 10^{-11}$ & NM \\
\hline 33 & 93.3 & 6.7 & 0.0 & $1.1 \times 10^{-11}$ & $3.3 \times 10^{-12}$ \\
\hline 34 & 91.8 & 2.8 & 5.5 & $4.6 \times 10^{-11}$ & $2.7 \times 10^{-11}$ \\
\hline 35 & 70.6 & 12.0 & 17.4 & $1.6 \times 10^{-11}$ & $3.6 \times 10^{-11}$ \\
\hline 36 & 80.7 & 19.2 & 0.1 & $1.3 \times 10^{-11}$ & $6.0 \times 10^{-11}$ \\
\hline 37 & 82.0 & 17.9 & 0.1 & $1.3 \times 10^{-11}$ & $2.4 \times 10^{-11}$ \\
\hline 38 & 77.6 & 10.8 & 11.7 & $1.5 \times 10^{-11}$ & $5.8 \times 10^{-11}$ \\
\hline 39 & 85.7 & 14.2 & 0.1 & $1.8 \times 10^{-11}$ & $2.7 \times 10^{-11}$ \\
\hline 40 & 85.4 & 14.5 & 0.1 & $3.3 \times 10^{-11}$ & $1.3 \times 10^{-10}$ \\
\hline 41 & 79.5 & 17.2 & 3.2 & $3.3 \times 10^{-11}$ & $1.1 \times 10^{-10}$ \\
\hline
\end{tabular}


Table SM4. Modeled relative contributions of $\mathrm{HO}^{\bullet}$ sources for the 41 cloud samples considering the new photolysis experimental rates in the model for $\mathrm{H}_{2} \mathrm{O}_{2}$, nitrite and nitrate and without iron chemistry. Modeled and experimental $\mathrm{R}_{\mathrm{HO}}^{\mathrm{f}}$ are also indicated. NM: not measured. 


\section{References}

Amels, T., Elias, H., Götz, U., Steingens, U., and Wannowius, K. J.: Kinetic investigation of the stability of peroxonitric acid and its reaction with sulfur(IV) in aqueous solution, in: Heterogeneous and liquid-phase processes, 1, 1996.

Behar, D., Czapski, G., and Duchovny, I.: Carbonate radical in flash photolysis and pulse radiolysis of aqueous carbonate solutions, J. Phys. Chem., 74, 2206-2210, 10.1021/j100909a029, 1970.

Benkelberg, H. J., Schäfer, A., and Warneck, P.: Product quantum yields for the photodecomposition of $\mathrm{H}_{2} \mathrm{O}_{2}$ and $\mathrm{Fe}(\mathrm{III})$ complexes in aqueous soltion in: Air Pollution research Report 33: Atmospheric Oxidation Processes, edited by: Becker, K. H., CEC, Brussels, 130, 1991.

Benkelberg, H. J., and Warneck, P.: Photodecomposition of iron(III) hydroxo and sulfato complexes in aqueous solution: Wavelength dependence of $\mathrm{OH}$ and $\mathrm{SO}_{4}^{-}$quantum yields, J. Phys. Chem., 99, 5214-5221, 10.1021/j100014a049, 1995.

Bielski, B. H. J., Cabelli, D. E., Arudi, R. L., and Ross, A. B.: Reactivity of $\mathrm{HO}_{2} / \mathrm{O}_{2}{ }^{-}$Radicals in Aqueous Solution, J. Phys.Chem. Ref. Data, 14, 1041-1100, 1985.

Bielski, B. H. J.: A pulse radiolysis study of the reaction of ozone with $\mathrm{Cl}_{2}$ in aqueous solutions, Rad. Phys. Chem., 41, 527-530, 1993.

Bjergbakke, E., Navaratnam, S., Parsons, B. J., and Swallow, A. J.: Reaction between hydroperoxo radicals and chlorine in aqueous solution, J. Am. Chem. Soc., 103, 5926-5928, 10.1021/ja00409a059, 1981.

Brandt, C., and van Eldik, R.: Transition metal-catalyzed oxidation of sulfur(IV) oxides. Atmosphericrelevant processes and mechanisms, Chem. Rev., 95, 119-190, 10.1021/cr00033a006, 1995.

Buxton, G. V., Malone, T. N., and Salmon, G. A.: Pulse radiolysis study of the reaction of $\mathrm{SO}_{5}{ }^{-}$with $\mathrm{HO}_{2}$, J. Chem. Soc., Faraday Transactions, 92, 1996a.

Buxton, G. V., McGowan, S., Salmon, G. A., Williams, J. E., and Wood, N. D.: A study of the spectra and reactivity of oxysulphur-radical anions involved in the chain oxidation of S(IV): A pulse and $\gamma$ radiolysis study, Atmospheric Environment, 30, 2483-2493, http://dx.doi.org/10.1016/13522310(95)00473-4, $1996 \mathrm{~b}$.

Buxton, G. V., Malone, T. N., and Salmon, G. A.: Reaction of $\mathrm{SO}_{4}{ }^{-}$with $\mathrm{Fe}^{2+}, \mathrm{Mn}^{2+}$ and $\mathrm{Cu}^{2+}$ in aqueous solution, J. Chem. Soc., Faraday Transactions, 93, 1997.

Buxton, G. V., Bydder, M., and Salmon, G. A.: The reactivity of chlorine atoms in aqueous solution. Part II. The equilibrium $\mathrm{SO}_{4}^{-}+\mathrm{Cl}^{-}=\mathrm{Cl}+\mathrm{SO}_{4}{ }^{2-}$, Phys. Chem. Chem. Phys., 1, 269-273, 1999.

Buxton, G. V., Bydder, M., Salmon, G. A., and Williams, J.: The reactivity of chlorine atoms in aqueous solution, Part III.The reactions of Cl with solutes, Phys. Chem. Chem. Phys., 2, 237-245, 2000.

Christensen, H., and Sehested, K.: Hydroperoxo and oxygen(1-) radicals at elevated temperatures, J. Phys. Chem., 92, 3007-3011, 10.1021/j100321a060, 1988.

Christensen, H., Sehested, K., and LÃ gager, T.: The reaction of hydrogen peroxide with Fe(II) ions at elevated temperatures, Rad. Phys. Chem., 41, 575-578, 1993.

Cotton, F. A., and Wilkinson, G.: Advanced inorganic chemistry: a comprehensive text, 4th ed., John Wiley \& Sons, New York, 1455 pp., 1980.

Damschen, D. E., and Martin, L. R.: Aqueous aerosol oxidation of nitrous acid by $\mathrm{O}_{2}, \mathrm{O}_{3}$, and $\mathrm{H}_{2} \mathrm{O}_{2}$, Atmos. Environ., 17, 2005-2011, 1983.

Das, T. N.: Reactivity and role of $\mathrm{SO}_{5}{ }^{-}$radical in aqueous medium chain oxidation of sulfite to sulfate and atmospheric sulfuric acid generation, J. Phys. Chem., 105, 9145-9155, 2001.

Draganic, Z. D., Negron-Mendoza, A., Sehested, K., Vujosevic, S. I., Navarro-Gonzales, R., AlbarranSanchez, M. G., and Draganic, I. G.: Radiolysis of aqueous solution of ammonium bicarbonate over a large dose range, Rad. Phys. Chem., 38, 317-321, 1991.

Eigen, M., Krause, W., Maash, H. G., and Demaeyer, L.: Rate constants of photolytic reactions in aqueous solution, in: Progress in reaction kinetics, edited by: Porter, G., Macmillan, N. J., 285-318, 1964.

Epstein, I. R., Kustin, K., and Simoyi, R. H.: Systematic design of chemical oscillators. 6. Nitrous acid decomposition catalyzed by an iron(II) complex: tris(3,4,7,8-tetramethyl-1,10phenanthroline)iron(II), J. Am. Chem. Soc., 104, 712-717, 10.1021/ja00367a013, 1982. 
Eriksen, T. E., Lind, J. A., and Merenyi, G.: On the acid-base equilibrium of the carbonate radical, Rad. Phys. Chem., 26, 197-199, 1985.

Exner, M., Herrmann, H., and Zellner, R.: Laser-based studies of reactions of the nitrate radical in aqueous solution, B. Buns. phys. Chem., 3, 470-477, 1992.

Faust, B. C., and Zepp, R. G.: Photochemistry of aqueous iron(III)-polycarboxylate complexes: roles in the chemistry of atmospheric and surface waters, Environ. Sci. Tech., 27, 2517-2522, 1993.

Gilbert, B. C., and Stell, J. K.: Mechanisms of peroxide decomposition. An ESR study of the reactions of the peroxomonosulphate anion $\left(\mathrm{HOOSO}_{3}^{-}\right)$with $\mathrm{Ti}(\mathrm{III}), \mathrm{Fe}(\mathrm{II})$, and alpha-oxygen-substituted radicals, J. Chem. Soc., Perkin Transactions 2, 1990.

Goldstein, S., and Czapski, G.: Reactivity of peroxynitric acid $\left(\mathrm{O}_{2} \mathrm{NOOH}\right)$ : A pulse radiolysis study, Inorg. Chem., 36, 4156-4162, 1997.

Graedel, T. E., and Weschler, C. J.: Chemistry within aqueous atmospheric aerosols and raindrops, Rev. Geophys., 19, 505-539, 10.1029/RG019i004p00505, 1981.

Graedel, T. E., and Goldberg, K. I.: Kinetic studies of raindrop chemistry 1. Inorganic and organic processes, J. Geophys. Res., 88, 10865-10882, 10.1029/JC088iC15p10865, 1983.

Grigor'ev, A. E., Makarov, I. E., and Pikaev, A. K.: Formation of $\mathrm{Cl}_{2}^{-}$in the bulk solution during the radiolysis of concentrated aqueous solutions of chloride, High Ener. Chem, 21, 1987.

Herrmann, H., Exner, M., and Zellner, R.: Reactivity trends in reactions of the nitrate radical $\left(\mathrm{NO}_{3}\right)$ with inorganic and organic cloudwater constituents, Geochim.Cosmochim. Acta, 58, 3239-3244, 1994.

Herrmann, H., and Zellner, R.: Reaction of $\mathrm{NO}_{3}$ radicals in aqueous solution, in: N-Centered Radicals, edited by: Alfassi, Z., Wiley, London, 291-343, 1998.

Hislop, K. A., and Bolton, J. R.: The photochemical generation of hydroxyl radicals in the UV-vis/Ferrioxalate/ $\mathrm{H}_{2} \mathrm{O}_{2}$ system, Environ. Sci.Tech., 33, 3119-3126, 1999.

Hoffmann, M. R., and Calvert, J. G.: Chemical transformation modules for Eulerian acid deposition models, Environmental Protection Agency, 1985.

Hoffmann, M. R.: On the kinetics and mechanism of oxidation of aquated sulfur dioxide by ozone, Atmos. Environ., 20, 1145-1154, 10.1016/0004-6981(86)90147-2, 1986.

Huie, R. E., and Clifton, C. L.: Temperature dependence of the rate constants for reactions of the sulfate radical, $\mathrm{SO}_{4}^{-}$, with anions, J. Phys. Chem., 94, 8561-8567, 1990.

Huie, R. E., Shoute, L., and Neta, P.: Temperature dependence of the rate constants for reactions of the carbonate radical with organic and inorganic reductants, Inter. J. Chem. Kin., 23, 541-542, 1991.

Jacobi, H. W., Herrmann, H., and Zellner, R.: Kinetic investigation of the $\mathrm{Cl}_{2}{ }^{-}$radical in the aqueous phase, in: Air Pollution research Report 57: Homogenous and heterogenous chemical processes in the troposphere, edited by: Becker, K. H., CEC, Brussels, 1996.

Jacobsen, F., Holcman, J., and Sehested, K.: Activation parameters of ferryl ion reactions in aqueous acid solutions, Inter. J. Chem. Kin., 29, 17-24, 10.1002/(sici)1097-4601(1997)29:1<17::aidkin3>3.0.co;2-o, 1997.

Jacobsen, F., Holcman, J., and Sehested, K.: Reactions of the ferryl ion with some compounds found in cloud water, Intern. J. Chem. Kin., 30, 215-221, 1998.

Jayson, G. G., Parsons, B. J., and Swallow, A. J.: Oxidation of ferrous ions by perhydroxyl radicals, J. Chem.Soc., Faraday Transactions, 69, 1973a.

Jayson, G. G., Parsons, B. J., and Swallow, A. J.: Appearance of sulphatoferric complexes in the oxidation of ferrous sulphate solutions. A study by pulse radiolysis, J. Chem. Soc., Faraday Transactions, 69, 1973b.

Jiang, P.-Y., Katsumura, Y., Nagaishi, R., Domae, M., Ishikawa, K., Ishigure, K., and Yoshida, Y.: Pulse radiolysis study of concentrated sulfuric acid solutions. Formation mechanism, yield and reactivity of sulfate radicals, J. Chem. Soc., Faraday Transactions, 88, 1653-1658, 1992.

Katsumura, Y.: $\mathrm{NO}_{2}$ and $\mathrm{NO}_{3}$ radicals in radiolysis of nitric acid solutions, in: $\mathrm{N}$ centered RAdical, edited by: Alfassi, Z. B., Wiley, Chichester, 393, 1998.

Kremer, M. L.: The Fenton reaction. Dependence of the rate on pH, J. Phys. Chem. A, 107, 1734-1741, 10.1021/jp020654p, 2003.

Lee, Y. N., and Lind, J. A.: Kinetics of aqueous oxidation of nitrogen (III) by hydrogen peroxide, J. Geophys. Res., 91, 2793-2800, 1986.

Lilie, R., Hanrahan, J., and Henglein, A.: O transfer reactions of the carbonate radical anion, Rad. Phys. Chem., 11, 225-227, 1978. 
Logager, T., Holcman, J., Sehested, K., and Pedersen, T.: Oxidation of ferrous ions by ozone in acidic solutions, Inorg. Chem., 31, 3523-3529, 10.1021/ic00043a009, 1992.

Logager, T., and Sehested, K.: Formation and decay of peroxynitric acid : a pulse radiolysis study, 39, American Chemical Society, Washington, DC, 10047-10052 pp., 1993.

Long, C. A., and Bielski, B. H. J.: Rate of reaction of superoxide radical with chloride-containing species, J. Phys. Chem., 84, 555-557, 10.1021/j100442a023, 1980.

Long, Y., Charbouillot, T., Brigante, M., Mailhot, G., Delort, A.-M., Chaumerliac, N., and Deguillaume, L.: Evaluation of modeled cloud chemistry mechanism against laboratory irradiation experiments: The $\mathrm{H}_{\mathrm{x}} \mathrm{O}_{\mathrm{y}} /$ iron/carboxylic acid chemical system, Atmos. Environ., 77, 686-695, 2013.

Maash, H. G.: Sulfur dioxide/water equilibrium between 0 degrees and 50 degrees Celcius, and examination data at low concentrations, in: Heterogenous Atmospheric Chemistry, Geophys. Monogr. Ser., edited by: Schryer, D. R., AGU, Washington, D. C., 187-1959, 1982.

Maßß, F., Elias, H., and Wannowius, K. J.: Kinetics of oxidation of hydrogen sulfite by hydrogen peroxide in aqueous solution: ionic strength e!ects and temperature dependence, Atmos. Environ., 33, 4413-4419, 1999.

Marsh, A. R. W., and McElroy, W. J.: The dissociation constant and Henry's law constant of $\mathrm{HCl}$ in aqueous solution, Atmos. Environ., 19, 1075-1080, 1985.

Maruthamuthu, P., and Neta, P.: Phosphate radicals. Spectra, acid-base equilibriums, and reactions with inorganic compounds, J. Phys. Chem., 82, 710-713, 10.1021/j100495a019, 1978.

Mertens, R., and Von Sonntag, C.: Photolysis $(\lambda=354 \mathrm{~nm})$ of tetrachloroethene in aqueous solutions, J. Photochem. Photobio: A chem., 85, 1-9, 1995.

Moorhead, E. G., and Sutin, N.: Rate and equilibrium constants for the formation of the monooxalate complex of iron(III), Inorg. Chem., 5, 1866-1871, 1966.

Nadtochenko, V. A., and Kiwi, J.: Photolysis of $\mathrm{FeOH}^{2+}$ and $\mathrm{FeCl}^{2+}$ in aqueous solution. Photodissociation kinetics and quantum yields, Inorg. Chem., 37, 5233-5238, 10.1021/ic9804723, 1998.

Padmaja, S., Neta, P., and Huie, R. E.: Rate constant for some reactions of inorganic radicals with inorganic ions. Temperature dependence and solvent dependence, J. Phys. Chem., 25, 445-455, 1993.

Park, J. Y., and Lee, Y. N.: Solubility and decomposition kinetics of nitrous acid in aqueous solution, J. Phys. Chem., 92, 6294-6302, 10.1021/j100333a025, 1988.

Pikaev, A. K., Sibirskaya, G. K., Shirshov, E. M., Glazunov, P. Y., and Spitsyn, V. I.: Pulse radiolysis of concentrated aqueous solutions of aqueous acid, Dokl. Phys. Chem. Proc. Acad. Sci., 215, 328-331, 1974.

Raabe, G.: Eine Laserphototyische Studie zur Kinetik der Reaktionen des $\mathrm{NO}_{3}$ Radicals in Wässriger Lösun, Cuvillier, Göttingen, Germany, 1996.

Riordan, E., Minogue, N., Healy, D., O'Driscol, P., and Sodeau, J. R.: Spectroscopic and optimization modeling study of nitrous acid in aqueous solution, J. Phys. Chem. A, 109, 779-786, 10.1021/jp040269v, 2005.

Ross, A. B., Bielski, B. H. J., Buxton, G. V., Greenstock, C. L., Helman, W. P., Huie, R. E., Grodkowski, J., Neta, P., and Mallard, W. G.: NDLR-NIST Solution kinetics database, version 3: NIST Standard reference database 40. National Institute of Standards and Technology, in, Gaithersburg, MD, 1994.

Rush, J. D., and Bielski, B. H. J.: Pulse radiolytic studies of the reactions of $\mathrm{HO}_{2} / \mathrm{O}_{2}{ }^{-}$with $\mathrm{Fe}(\mathrm{II}) / \mathrm{Fe}(\mathrm{III})$ ions. The reactivity of $\mathrm{HO}_{2} / \mathrm{O}_{2}^{-}$with ferric ions and its implication on the occurrence of the HaberWeiss reaction, J. Phys. Chem., 89, 5062-5066 1985.

Sehested, K., Holcman, J., and Hart, E. J.: Rate constants and products of the reactions of $\mathrm{e}_{\mathrm{aq}}{ }^{-} \mathrm{O}_{2}{ }^{-}$and $\mathrm{H}$ with ozone in aqueous solutions, J. Phys. Chem., 87, 1951-1954, 10.1021/j100234a024, 1983.

Sehested, K., Holcman, J., Bjergbakke, E., and Hart, E. J.: A pulse radiolytic study of the reaction hydroxyl + ozone in aqueous medium, J. Phys. Chem., 88, 4144-4147, 10.1021/j150662a058, 1984.

Sehested, K., Logager, T., Holoman, J., and Nielsen, O. J.: Formation and reactions of the $\mathrm{NO}_{3}$ radical in aqueous solution, in: Proceedings of EUROTRAC Symposium '94: Transport and Chemical Transformation of Pollutants in the Troposphere, edited by: Borrell, P. M., Cvitas, T., and Seiler, W., SPB Academic Publishing, The Hague, 999-1004, 1994.

Smith, R. M., and Martell, A. E.: Critical Stability Constants, Volume 4: Inorganic Complexes, Plenum, New York, 1976. 
Tang, Y., Thorn, R. P., Mauldin Iii, R. L., and Wine, P. H.: Kinetics and spectroscopy of the $\mathrm{SO}_{4}{ }^{-}$radical in aqueous solution, J. Photochem. Photobiol. Chem A, 44, 243-258, http://dx.doi.org/10.1016/10106030(88)80097-2, 1988.

Thornton, A. T., and Laurence, G. S.: Kinetics of oxidation of transition-metal ions by halogen radical anions. Part I. The oxidation of iron(II) by dibromide and dichloride ions generated by flash photolysis, J. Chem. Soc. Dalton Trans., 1973.

Wang, T. X., and Margerum, D. W.: Kinetics of Reversible Chlorine Hydrolysis: Temperature Dependence and General-Acid/Base-Assisted Mechanisms, Inorg. Chem., 33, 1050-1055, 10.1021/ic00084a014, 1994.

Weschler, C. J., Mandich, M. L., and Graedel, T. E.: Speciation, photosensitivity, and reactions of transition metal Ions in atmospheric droplets, J. Geophys. Res., 91, 5189-5204, 10.1029/JD091iD04p05189, 1986.

Williams, J.: PhD-Thesis, University of Leeds, 1996.

Xu, F. C., Krouse, H. R., and Swaddle, T. W.: Conjugate base pathway for water exchange on aqueous chromium(III): variable-pressure and -temperature kinetic study, Inorg. Chem., 24, 267-270, 10.1021/ic00197a005, 1985.

Yu, X. Y., and Barker, J. R.: Hydrogen peroxide photolysis in acidic aqueous solutions containing chloride ions. I. Chemical Mechanism, J. Phys. Chem. A, 107, 1313-1324, 2003.

$\mathrm{Yu}, \mathrm{X}$. Y.: Critical evaluation of rate constants and equilibrium constants of hydrogen peroxide photolysis in acidic aqueous solutions containing chloride ions, J. Phys. Chem. Ref. Data, 33, 2004.

Zellner, R., Exner, M., and Herrmann, H.: Absolute $\mathrm{OH}$ quantum yields in the laser photolysis of nitrate, nitrite and dissolved $\mathrm{H}_{2} \mathrm{O}_{2}$ at 308 and $351 \mathrm{~nm}$ in the temperature range 278-353 K, J. Atmos. Chem., 10, 411-425, 10.1007/bf00115783, 1990.

Ziajka, J., Beer, F., and Warneck, P.: Iron-catalysed oxidation of bisulphite aqueous solution: Evidence for a free radical chain mechanism, Atmos. Environ., 28, 2549-2552, 10.1016/1352-2310(94)904057, 1994. 